\title{
Realizations of the Monster Lie Algebra
}

\author{
Elizabeth Jurisich* \\ James Lepowsky \\ Robert L. Wilson
}

\author{
Department of Mathematics, Rutgers University \\ New Brunswick, NJ 08903 \\ ${ }^{*}$ Current address: Department of Mathematics, University of Chicago \\ Chicago, IL 60637
}

e-mail addresses: jurisich@math.rutgers.edu, lepowsky@math.rutgers.edu, rwilson@math.rutgers.edu

\section{Introduction}

In this paper we reinterpret the theory of generalized Kac-Moody Lie algebras in terms of local Lie algebras formed from reductive or Kac-Moody algebras and certain modules (possibly infinite-dimensional) for these algebras. We exploit the fact, established in [19], that certain generalized Kac-Moody algebras contain specific large free subalgebras, in order to exhibit these generalized KacMoody algebras as explicitly prescribed Lie algebras of operators acting on tensor algebras. In the most important special case, that of R. Borcherds' Monster Lie algebra $\mathfrak{m}$, introduced in [4] (see also [5]), we apply the free subalgebra result [19] to simplify Borcherds' work [4] on the Conway-Norton conjectures (see [8]) for the "moonshine module" $V^{\natural}([11]$, [12]) for the Fischer-Griess Monster group $M$. In particular, we realize $\mathfrak{m}$ as an explicitly prescribed $M$-covariant Lie algebra of operators acting on the tensor algebra over a certain $\mathfrak{g l}_{2^{-}}$and $M$-module built in a simple way from the $M$-module $V^{\natural}$.

In [4], Borcherds produces recursion relations which, along with initial conditions, uniquely characterize the McKay-Thompson series (i.e., the graded traces) of the elements of $M$ acting on the infinite-dimensional $M$-module $V^{\natural}$ constructed in [11], [12]. In this way he completes the proof of the Conway-Norton conjectures as they relate to $V^{\natural}$, in the sense that he shows that the McKayThompson series for $V^{\natural}$ do indeed agree with the modular functions listed in [8] for all elements of $M$, since the coefficients of those modular functions satisfy the same recursion relations (replication formulas) and initial conditions. For instance, for the identity element of $M$ the corresponding McKay-Thompson 
series is the modular function $J(q)=j(q)-744$, which is the graded dimension of $V^{\natural}$. (This is one of the McKay-Thompson series for $V^{\natural}$ already determined in [11], [12].) The proof in [4] involves defining the Lie algebra $\mathfrak{m}$, establishing a triangular decomposition $\mathfrak{m}=\mathfrak{n}^{+} \oplus \mathfrak{h} \oplus \mathfrak{n}^{-}$and a denominator identity for $\mathfrak{m}$, and computing the homology of the subalgebra $\mathfrak{n}^{+} \subset \mathfrak{m}$ (or equivalently, of the subalgebra $\mathfrak{n}^{-}$). Borcherds is then able to show that the coefficients of the McKay-Thompson series satisfy certain recursion relations. As we will explain below, much of the theory involved in the proof in [4] is illuminated if we decompose $\mathfrak{m}$ as

$$
\mathfrak{m}=\mathfrak{u}^{+} \oplus \mathfrak{g l}_{2} \oplus \mathfrak{u}^{-},
$$

where $\mathfrak{u}^{+}$and $\mathfrak{u}^{-}$are the Lie algebras proved to be free in [19], and if we consider the $\mathfrak{g l}_{2}$-module $\mathfrak{u}^{-}$in place of $\mathfrak{n}^{-}$. We base our considerations on this decomposition rather than the triangular decomposition used in [4].

The properties of the Monster Lie algebra are of central importance to the proof in [4]. In Section 2 we recall facts about generalized Kac-Moody algebras, of which $\mathfrak{m}$ is an example. Borcherds' original work on the subject [2] contains results such as character formulas and a denominator identity for generalized Kac-Moody algebras. V. Kac in [21] gives an outline (without detail) of how to rigorously develop the theory of generalized Kac-Moody algebras by indicating that one should follow the arguments presented there for Kac-Moody algebras; see also [17]. Included in [18] is an exposition developing the theory of generalized Kac-Moody algebras, including an extension of the homology results of [14] (not covered in [21]) to these more general Lie algebras. That this can be done is mentioned and used in [4]. This homology theory gives another proof of the character and denominator formulas (see [18]). We find it appropriate to work with the extended Lie algebra as in [14] and [24] (that is, the Lie algebra with suitable degree derivations adjoined). Equivalently, one can generalize the theorems in [21]. In either of these approaches the Cartan subalgebra is sufficiently enlarged to make the simple roots linearly independent and of multiplicity one.

It is shown in [19] that any generalized Kac-Moody algebra $\mathfrak{g}$ that has no mutually orthogonal imaginary simple roots can be decomposed as $\mathfrak{g}=\mathfrak{u}^{+} \oplus$ $\left(\mathfrak{g}_{S}+\mathfrak{h}\right) \oplus \mathfrak{u}^{-}$where $\mathfrak{g}_{S}$ is a (semisimple or) Kac-Moody Lie algebra and $\mathfrak{u}^{+}$and $\mathfrak{u}^{-}$are free Lie algebras over particular modules for $\mathfrak{g}_{S}$. This theorem is recalled in Section 2 below, where we also summarize properties of $\mathfrak{m}$ which we use. It is also shown that there are Lie algebras which cover $\mathfrak{m}$ such that the natural action of $M$ on $\mathfrak{m}$ lifts to an action of $M$ on these larger generalized Kac-Moody algebras.

Facts about local Lie algebras that we use are also included in Section 2. Mainly, we recall that the maximal Lie algebra $\mathfrak{a}_{\max }$ associated to a local Lie algebra $\mathfrak{a}=\mathfrak{a}_{1} \oplus \mathfrak{a}_{0} \oplus \mathfrak{a}_{-1}$ is such that $\mathfrak{a}_{\text {max }}^{ \pm}$is the free Lie algebra on $\mathfrak{a}_{ \pm 1}$, as in [20]. In light of the results of [19] mentioned above, it is not surprising that these generalized Kac-Moody algebras with suitable derivations adjoined are closely related to maximal Lie algebras $\mathfrak{a}_{\max }$ associated to the appropriate 
local Lie algebras $\mathfrak{a}$. We show this in Section 3.

In Section 4, we discuss some examples of standard (irreducible) modules which have the special property that they are induced from irreducible modules of the subalgebra $\left(\mathfrak{g}_{S}+\mathfrak{h}\right) \oplus \mathfrak{u}^{+}$. As mentioned above, combined with the free subalgebra result, this gives yet another natural realization of the Monster Lie algebra, this time as an explicitly prescribed $M$-covariant Lie algebra of operators on the space $T(\mathcal{V})$, where $\mathcal{V}$ is one of the two (infinite-dimensional) $\mathfrak{g l}_{2^{-}}$and $M$-modules $\mathfrak{a}_{ \pm 1}$ occurring in the local Lie algebra construction (here $\mathfrak{a}_{0}=\mathfrak{g l}_{2}$ ). The examples of standard modules that we consider in this paper are all "generalized Verma modules," that is, they are induced from irreducible modules for "parabolic subalgebras" (e.g., the subalgebra $\mathfrak{p}=\left(\mathfrak{g}_{S}+\mathfrak{h}\right) \oplus \mathfrak{u}^{+}$for a generalized Kac-Moody algebra $\left.\mathfrak{g}=\mathfrak{u}^{-} \oplus\left(\mathfrak{g}_{S}+\mathfrak{h}\right) \oplus \mathfrak{u}^{+}\right)$. The considerations on generalized Verma modules in [14] and [25] remain applicable to the case of generalized Kac-Moody algebras. If we choose the right parabolic subalgebra and highest weight $\lambda$, then the corresponding generalized Verma module is a standard module, and in particular, it is irreducible. In the case where $\mathfrak{u}^{-}$is a free Lie algebra over a vector space $V$, the tensor algebra over $V$ clearly has the structure of a generalized Verma module (see Section 4). As we have mentioned, this includes the case of the Monster Lie algebra $\mathfrak{m}$. The "fundamental modules" constructed in [15] for those generalized Kac-Moody algebras (or Borcherds algebras, as they are called in [15]) with one simple imaginary root are special cases of these induced modules. For the case of $\mathfrak{m}$, we conjecture that the weight-zero components $v_{0}$ of the vertex operators $Y(v, z)$ for $v \in \mathcal{V}$ generate a free associative algebra of operators on $\mathfrak{m}$. This may provide a more conceptual framework for studying the irreducible $\mathfrak{m}$-modules $T(\mathcal{V})$. (See Sections 2 and 4.)

Finally, in Section 5, we use the free Lie algebra structure of $\mathfrak{u}^{-}$to simplify part of the proof appearing in [4] concerning the replication formulas. We follow the general ideas of Borcherds' proof, but we use the subalgebra $\mathfrak{u}^{-}$ in place of $\mathfrak{n}^{-}$. Because of its structure as a free Lie algebra, computations involving $\mathfrak{u}^{-}$are comparatively simple. For example, the homology of $\mathfrak{u}^{-}$with coefficients in the trivial module is elementary to compute, while providing us with essentially the same information as the homology of $\mathfrak{n}^{-}$. Moreover, the formula, obtained from the Euler-Poincaré identity, that leads to replication (now symmetric in the formal variables $p$ and $q$ ) is visibly similar to formulas occurring in such classical references on free Lie algebras as [6] and [28] (as is the proof of the denominator identities in [19]). We are able to obtain the replication formula as it appears in [27] by carrying out an analog of a computation in [6]. After proving a Möbius inversion formula consistent with Adams operations, we establish recursion relations equivalent to replication. Even though replication is more easily understood in this context, it is still not clear why series satisfying these replication formulas should automatically have modular transformation properties. Philosophically, the fact that $\mathfrak{n}^{+}\left(\right.$or $\left.\mathfrak{n}^{-}\right)$contains such a large free Lie algebra explains why computations such as determining root multiplicities 
and the replication formulas are at all manageable for $\mathfrak{m}$.

This paper was presented by E. J. and J. L. at the June, 1994 AMS-IMSSIAM Joint Summer Research Conference on Moonshine, the Monster and Related Topics at Mount Holyoke College.

We are grateful to S.-J. Kang for informing us that formula (37) in this paper has been independently obtained in joint work with V. Kac.

We would like to thank R. Borcherds, F. Knop and C. Weibel for helpful comments. We also thank R. Gebert and J. Teschner, K. Harada, M. Miyamoto and H. Yamada, S. J. Kang and S. Naito for sending us preprints of their works on generalized Kac-Moody algebras. E. J. is supported in part by a Rutgers University Louis Bevier Graduate Fellowship, and J. L. and R. W. are supported in part by NSF grant DMS-9111945.

\section{Preliminary Material}

\section{$2.1 \quad$ Notation}

For any vector space $V$ we let $L(V)$ denote the free Lie algebra over $V$, and $T(V)$ the tensor algebra over $V$. Recall that $T(V)$ is the universal enveloping algebra of $L(V)$. Let $V^{*}$ denote the dual space of $V$. For a $\mathbb{Z}$-graded vector space $V=\coprod_{i \in \mathbb{Z}} V_{i}$ we let $V^{ \pm}$denote $\coprod_{i \in \pm \mathbb{Z}_{+}} V_{i}$. Unless otherwise indicated, vector spaces will be over $\mathbb{C}$. We denote the Monster group by $M$ and the "moonshine module" for $M$ by $V^{\natural}$, as in [12].

\subsection{Generalized Kac-Moody algebras}

The theory of generalized Kac-Moody Lie algebras with imaginary simple roots was developed by Borcherds starting in [2]. Two further definitions (different from the first and from one another) are given in [3] and [4]. Of these definitions the one given below most resembles the one appearing in [2], where there is a definition in terms of an index set $I$, a vector space $H$, a set of generators $H \cup\left\{e_{i}, f_{i} \mid i \in I\right\}$, and relations, determined by square matrices, which agree with the ones given here. Borcherds does not divide out by the radical (that is, the largest graded ideal having trivial intersection with $\mathfrak{h}$ ) in his definition of generalized Kac-Moody algebra. In [3] Borcherds extends his definition to include some additional elements $h_{i j}$ which are central. These elements do not enter into our discussion. In [4] Borcherds defines generalized Kac-Moody algebras in terms of "an almost positive definite bilinear form" and an involution, and it is a theorem stated in [4] (see also [17] and [19] for clarification of the statement and for two different detailed proofs; cf. [21]) that such Lie algebras are closely related to Lie algebras defined from matrices.

The definition given here of the generalized Kac-Moody algebra $\mathfrak{g}(A)$ (see below) is the same as that in [19]. In [18] and [21] a generalized Kac-Moody 
algebra is defined as $\mathfrak{g}(A)$ modulo its radical. The radical of $\mathfrak{g}(A)$ is in fact zero (see [17] and [18] for detailed proofs, clarifying [2], using [13] or [21]; or see [19], where there is a relatively elementary argument for the case of Lie algebras satisfying the hypothesis of Theorem 2.1 below-which are the main examples discussed in this paper - showing that the vanishing of the radical for Kac-Moody algebras implies the vanishing of the radical for this class). Thus the definition given here is equivalent to those in [18] and [21].

Theorem 2.1, given at the end of this section, is the main theorem of [19], and is used throughout this paper.

We will use the notation of [14], [24] and [25], suitably generalized to the case of generalized Kac-Moody Lie algebras in [18]. We restrict our attention to the case of symmetric matrices (although symmetrizable matrices could be easily handled) and countable index sets.

Let $I$ be a (finite or) countable index set and let $A=\left(a_{i j}\right)_{i, j \in I}$ be a matrix with entries in $\mathbb{R}$, satisfying the following conditions:

(C1) $A$ is symmetric.

(C2) If $i \neq j(i, j \in I)$, then $a_{i j} \leq 0$.

(C3) If $a_{i i}>0(i \in I)$, then $\frac{2 a_{i j}}{a_{i i}} \in \mathbb{Z}$ for all $j \in I$.

Let $\mathfrak{g}(A)$ be the Lie algebra with generators $h_{i}, e_{i}, f_{i}, i \in I$, and the following defining relations: For all $i, j, k \in I$,

(R1) $\left[h_{i}, h_{j}\right]=0$,

(R2) $\left[h_{i}, e_{k}\right]-a_{i k} e_{k}=0$,

(R3) $\left[h_{i}, f_{k}\right]+a_{i k} f_{k}=0$,

(R4) $\left[e_{i}, f_{j}\right]-\delta_{i j} h_{i}=0$,

(R5) $\left(\operatorname{ad} e_{i}\right)^{\frac{-2 a_{i j}}{a_{i i}}+1} e_{j}=0$ and $\left(\operatorname{ad} f_{i}\right)^{\frac{-2 a_{i j}}{a_{i i}}+1} f_{j}=0$ for all $i \neq j$ with $a_{i i}>0$,

(R6) $\left[e_{i}, e_{j}\right]=0$ and $\left[f_{i}, f_{j}\right]=0$ whenever $a_{i j}=0$.

Definition: The Lie algebra $\mathfrak{g}(A)$ is the generalized Kac-Moody (Lie) algebra associated to the matrix $A$. Any Lie algebra of the form $\mathfrak{g}(A) / \mathfrak{c}$ where $\mathfrak{c}$ is a central ideal is called a generalized Kac-Moody algebra.

In $\mathfrak{g}(A)$, the elements $h_{i}, e_{i}, f_{i}$ for $i \in I$ are linearly independent. Define the Cartan subalgebra $\mathfrak{h}$ of $\mathfrak{g}(A)$ to be the span of the $h_{i}$ for $i \in I$. The center of $\mathfrak{g}(A)$ lies in $\mathfrak{h}$.

Let $A$ satisfy $(C 1)-(C 3)$ and let $S \subset I$ be the set of all indices $i$ such that $a_{i i}>0$. (Many of the following considerations apply for more general subsets of $I$; it is for convenience that we fix $S$ as indicated.) Let $B$ be the the submatrix of $A$ given by $\left(a_{i j}\right)_{i, j \in S}$. 
The associated Lie algebra $\mathfrak{g}(B)$ is a Kac-Moody algebra (with a symmetric defining matrix). Let $\mathfrak{g}_{S}$ be the Lie subalgebra of $\mathfrak{g}(A)$ generated by $\left\{e_{i}, h_{i}, f_{i}\right\}_{i \in S}$. Then $\mathfrak{g}_{S}$ is isomorphic to $\mathfrak{g}(B)$. Note that we are using the characterization of $\mathfrak{g}(B)$ in terms of generators and relations, i.e., we are using the fact that the radical is zero (see [13] or [21]; this is Serre's theorem [29] in case $B$ is of finite type). For the important case of the Monster Lie algebra $\mathfrak{m}$, described in more detail in the next section, $B=(2)$ and $\mathfrak{g}_{S} \cong \mathfrak{s l}_{2}$.

The Lie algebra $\mathfrak{g}=\mathfrak{g}(A)$ is naturally graded by $\mathbb{Z}^{I}$. Let $D_{i}$ be the $i^{\text {th }}$ degree derivation of $\mathfrak{g}$ with respect to this grading, i.e., $D_{i} e_{j}=\delta_{i j} e_{j}, D_{i} f_{j}=-\delta_{i j} f_{j}$ and $D_{i} h_{j}=0$ for all $i, j \in I$. The span of the $D_{i}$ for $i \in I$ forms an abelian Lie algebra $\mathfrak{d}_{0}$ of derivations of $\mathfrak{g}$. Given a subspace $\mathfrak{d}$ of $\mathfrak{d}_{0}$ let $\mathfrak{g}^{e}$ be the semidirect product $\mathfrak{d} \ltimes \mathfrak{g}$. Denote by $\mathfrak{h}^{e}$ the abelian subalgebra $\mathfrak{d} \oplus \mathfrak{h}$. The Lie algebra $\mathfrak{g}^{e}$ is called the extended Lie algebra. Call $\mathfrak{h}^{e}$ the Cartan subalgebra of $\mathfrak{g}^{e}$.

Define the simple roots $\alpha_{i} \in\left(\mathfrak{h}^{e}\right)^{*}$ for $i \in I$ by the conditions $\left[h, e_{i}\right]=\alpha_{i}(h) e_{i}$ for all $h \in\left(\mathfrak{h}^{e}\right)^{*}, i \in I$. Fix a subspace $\mathfrak{d}$ of $\mathfrak{d}_{0}$ such that the simple roots $\alpha_{i}$ are linearly independent. There is a unique symmetric bilinear form $(\cdot, \cdot)$ on the span of the $\alpha_{i}, i \in I$, determined by the conditions $\left(\alpha_{i}, \alpha_{j}\right)=a_{i j}$. This can be extended (not necessarily uniquely) to a symmetric bilinear form, which we continue to call $(\cdot, \cdot)$, on all of $\left(\mathfrak{h}^{e}\right)^{*}$ such that $\left(\varphi, \alpha_{i}\right)=\varphi\left(h_{i}\right)$ for all $\varphi \in\left(\mathfrak{h}^{e}\right)^{*}$.

For $\alpha \in\left(\mathfrak{h}^{e}\right)^{*}$ define

$$
\mathfrak{g}^{\alpha}=\left\{x \in \mathfrak{g} \mid[h, x]=\alpha(h) x \text { for all } h \in \mathfrak{h}^{e}\right\} .
$$

Nonzero elements $\alpha \in\left(\mathfrak{h}^{e}\right)^{*}$ such that $\mathfrak{g}^{\alpha} \neq 0$ are called roots. Let $\Delta \subset\left(\mathfrak{h}^{e}\right)^{*}$ be the set of roots, and $\Delta_{+}$the set of roots which are nonnegative integral linear combinations of $\alpha_{i}$ 's. The roots in $\Delta_{+}$are called positive roots. Let $\Delta_{-}=-\Delta_{+}$, the set of negative roots. All of the roots are either positive or negative. There is a root space decomposition

$$
\mathfrak{g}=\coprod_{\varphi \in \Delta_{+}} \mathfrak{g}^{\varphi} \oplus \mathfrak{h} \oplus \coprod_{\varphi \in \Delta_{-}} \mathfrak{g}^{\varphi} .
$$

Define the set of real roots $\Delta_{R}$ to be the set of $\alpha \in \Delta$ such that $(\alpha, \alpha)>0$. The set of imaginary roots is $\Delta \backslash \Delta_{R}$. The imaginary simple roots are those $\alpha_{i}$ for which $a_{i i} \leq 0$.

The symmetric bilinear form on the span of the $\alpha_{i}, i \in I$, can be transferred to $\mathfrak{h}$ by letting $\left(h_{i}, h_{j}\right)_{\mathfrak{h}}=\left(\alpha_{i}, \alpha_{j}\right)=a_{i j}$. This form can be extended (not uniquely, for it depends upon a choice of symmetric bilinear form on $\mathfrak{d})$ to a symmetric bilinear form $(\cdot, \cdot)_{\mathfrak{h}^{e}}$ on $\mathfrak{h}^{e}=\mathfrak{d} \oplus \mathfrak{h}$, satisfying the conditions $\left(d, h_{j}\right)=$ $\alpha_{j}(d)$ for $d \in \mathfrak{d}$ and $j \in I$. We assume that this form on $\mathfrak{h}^{e}$ is consistent with the form on $\left(\mathfrak{h}^{e}\right)^{*}$. There is a unique invariant symmetric bilinear form $(\cdot, \cdot)_{\mathfrak{g}^{e}}$ on $\mathfrak{g}^{e}$ which extends $(\cdot, \cdot)_{\mathfrak{h}}$. This form satisfies the condition

$$
\left(\mathfrak{g}^{\varphi}, \mathfrak{g}^{\psi}\right)_{\mathfrak{g}^{e}}=\left(\mathfrak{h}^{e}, g^{\varphi}\right)_{\mathfrak{g}^{e}}=0
$$


for all $\varphi, \psi \in \Delta$ such that $\varphi+\psi \neq 0$. Given any $\varphi=\sum_{i \in I} n_{i} \alpha_{i} \in \Delta\left(n_{i} \in \mathbb{Z}\right)$, we define $h_{\varphi}=\sum_{i \in I} n_{i} h_{i}$. Then for $a \in \mathfrak{g}^{\varphi}, b \in \mathfrak{g}^{-\varphi}$, the form $(\cdot, \cdot)_{\mathfrak{g}^{e}}$ satisfies the condition

$$
[a, b]=(a, b) h_{\varphi}
$$

In particular, $\left(e_{i}, f_{j}\right)_{\mathfrak{g}^{e}}=\delta_{i j}$ for $i, j \in I$. (See [18] for details; cf. [20], [24], [26].)

We assume that in the case in which $A$ is a (finite) generalized Cartan matrix, the space $\mathfrak{d}$ and the form on $\mathfrak{d}$ are chosen so that the form $(\cdot, \cdot)_{\mathfrak{h}}$ on the (finite-dimensional) space $\mathfrak{h}^{e}$ is nonsingular, so that the Lie algebra $\mathfrak{g}^{e}=\mathfrak{g}(A)^{e}$ is, in this case, the usual Kac-Moody algebra (cf. [21]) and the form $(\cdot, \cdot)_{\mathfrak{g}^{e}}$ is nondegenerate.

The center of the unextended Lie algebra $\mathfrak{g}$ is equal to the radical of the form $(\cdot, \cdot)$ restricted to $\mathfrak{g}$. We also note that any element of the center $h=\sum_{j \in I} c_{j} h_{j}$ $\left(c_{j} \in \mathbb{C}, c_{j}=0\right.$ for almost all $\left.j \in I\right)$ provides a linear dependence relation $\sum_{j \in I} c_{j} a_{i j}$, among the columns of the matrix $A$ and conversely, any such relation among columns of $A$ determines an element of the center of $\mathfrak{g}$.

We make the following definitions: $\Delta^{S}=\Delta \cap \coprod_{i \in S} \mathbb{Z} \alpha_{i}, \Delta_{+}^{S}=\Delta_{+} \cap \Delta^{S}$ and $\Delta_{-}^{S}=\Delta_{-} \cap \Delta^{S}$. Denote by $\mathfrak{h}_{S}$ the span of the $h_{i}, i \in S$. Then we have the root space decomposition:

$$
\mathfrak{g}_{S}=\coprod_{\varphi \in \Delta_{+}^{S}} \mathfrak{g}^{\varphi} \oplus \mathfrak{h}_{S} \oplus \coprod_{\varphi \in \Delta_{-}^{S}} \mathfrak{g}^{\varphi} .
$$

Define the following subalgebras of $\mathfrak{g}=\mathfrak{g}(A)$ :

$$
\begin{gathered}
\mathfrak{n}^{+}=\coprod_{\varphi \in \Delta_{+}} \mathfrak{g}^{\varphi} ; \quad \mathfrak{n}^{-}=\coprod_{\varphi \in \Delta_{-}} \mathfrak{g}^{\varphi} ; \quad \mathfrak{n}_{S}^{+}=\coprod_{\varphi \in \Delta_{+}^{S}} \mathfrak{g}^{\varphi} ; \\
\mathfrak{n}_{S}^{-}=\coprod_{\varphi \in \Delta_{-}^{S}} \mathfrak{g}^{\varphi} ; \quad \mathfrak{u}^{+}=\coprod_{\varphi \in \Delta_{+} \backslash \Delta_{+}^{S}} \mathfrak{g}^{\varphi} ; \quad \mathfrak{u}^{-}=\coprod_{\varphi \in \Delta_{-} \backslash \Delta_{-}^{S}} \mathfrak{g}^{\varphi} ; \quad \mathfrak{r}=\mathfrak{g}_{S}+\mathfrak{h} .
\end{gathered}
$$

Let $\mathfrak{p}=\mathfrak{r} \oplus \mathfrak{u}^{+}$, the "parabolic subalgebra" of $\mathfrak{g}$ defined by $S$. Note that

$$
\mathfrak{g}=\mathfrak{u}^{-} \oplus \mathfrak{p}
$$

The subalgebras $\mathfrak{p}^{e}, \mathfrak{r}^{e}$ and $\mathfrak{g}_{S}^{e}$ are defined in the obvious ways.

Let $X$ be an $\mathfrak{h}^{e}$-module, e.g., a $\mathfrak{g}^{e}$-module considered as an $\mathfrak{h}^{e}$-module by restriction, and let $\nu \in\left(\mathfrak{h}^{e}\right)^{*}$. Define the corresponding weight space

$$
X_{\nu}=\left\{x \in X \mid h \cdot x=\nu(h) x \text { for all } h \in \mathfrak{h}^{e}\right\} .
$$

We say that $\nu$ is a weight if $X_{\nu} \neq 0$ and that the nonzero elements of $X_{\nu}$ are weight vectors of weight $\nu$. The module $X$ is called a weight module if $X$ is a direct sum of its weight spaces.

A $\mathfrak{g}^{e}$-module is called a highest weight module if it is generated by a weight vector $x$ annihilated by $\mathfrak{n}^{+}$. The weight of $x$ is called the highest weight of 
$X$. For any highest weight module $X$ we have $U\left(\mathfrak{n}^{-}\right) \cdot x=X$. Lowest weight modules are defined analogously.

Concepts defined above for $\mathfrak{g}(A)$ such as extended Lie algebra, roots, simple roots, Cartan subalgebra and weights can be defined analogously for a generalized Kac-Moody algebra of the form $\mathfrak{g}(A) / \mathfrak{c}$ with $\mathfrak{c}$ a subspace of the center. Note that in particular, the roots of $\mathfrak{g}(A)$, which are elements of $\left(\mathfrak{h}^{e}\right)^{*}$, vanish on $\mathfrak{c} \subset \mathfrak{h}$ and may therefore be identified with the roots of $\mathfrak{g}(A) / \mathfrak{c}$, which are elements of the dual of $(\mathfrak{h} / \mathfrak{c})^{e}=\mathfrak{d} \oplus \mathfrak{h} / \mathfrak{c}$. We shall thereby identify the roots, simple roots, the $e_{i}$ 's and the $f_{i}$ 's (but not the $h_{i}$ 's), etc., of $\mathfrak{g}(A)$ with those of $\mathfrak{g}(A) / \mathfrak{c}$. The simple roots remain linearly independent. For those $\mathfrak{g}(A)^{e}$ weight modules $X$ on which $\mathfrak{c}$ acts trivially, $X$ is also a $(\mathfrak{g}(A) / \mathfrak{c})^{e}$-module, and we identify the weights, etc., for $X$ as $\mathfrak{g}(A)^{e}$ and $(\mathfrak{g}(A) / \mathfrak{c})^{e}$-modules.

We shall use the following theorem from [19]:

Theorem 2.1 Let $\mathfrak{g}(A)=\mathfrak{g}$ be a generalized Kac-Moody algebra such that if $\alpha_{i}$ and $\alpha_{j}$ are two distinct imaginary simple roots then $a_{i j}<0$. Let $S=\{i \in$ $\left.I \mid a_{i i}>0\right\}$. Then $\mathfrak{g}=\mathfrak{u}^{+} \oplus \mathfrak{r} \oplus \mathfrak{u}^{-}$, where $\mathfrak{u}^{-}$is the free Lie algebra on the direct sum of the integrable highest weight $\mathfrak{g}_{S}$-modules $U\left(\mathfrak{n}_{S}^{-}\right) \cdot f_{j}$ for $j \in I \backslash S$ and $\mathfrak{u}^{+}$is the free Lie algebra on the direct sum of the integrable lowest weight $\mathfrak{g}_{S}$-modules $U\left(\mathfrak{n}_{S}^{+}\right) \cdot e_{j}$ for $j \in I \backslash S$.

\subsection{The Monster Lie algebra}

The Monster Lie algebra $\mathfrak{m}$ is defined by Borcherds in [4] (see [19] for more detail and further references). It is constructed using the vertex operator algebra $V^{\natural}$ and the vertex algebra $V_{L}$ associated to an even Lorentzian lattice $L$ of rank 2 , which we identify with $\mathbb{Z} \oplus \mathbb{Z}$, equipped with the bilinear form $\langle\cdot, \cdot\rangle$ given by the matrix $\left(\begin{array}{cc}0 & -1 \\ -1 & 0\end{array}\right)$. The Lie algebra $\mathfrak{m}$ is a certain quotient of the "physical subspace" $P_{1}$ of the vertex algebra $V^{\natural} \otimes V_{L}\left(P_{1}\right.$ is the space of lowest weight vectors of weight one for the Virasoro algebra): $\mathfrak{m}=P_{1} / R$, where $R$ is the radical of a natural symmetric bilinear form. We do not need the details of the actual definition in this paper, but will quote results from [4], [12] and [19] as they are needed. (We note that Borcherds' construction of the Lie algebra is over $\mathbb{R}$, while we are working over $\mathbb{C}$.)

The vertex operator algebra $V^{\natural}$ is equipped with an action of $M$, and $V^{\natural}$ has a natural $M$-invariant symmetric nondegenerate bilinear form (see [12]).

We grade $V^{\natural}$ as $\bigsqcup_{i \geq-1} V_{i}^{\natural}$ where $V_{i}^{\natural}$ is the span of all vectors of conformal weight $i+1$. Recall ([11], [12]) that $V^{\natural}$ has graded dimension $\sum_{i \geq-1}\left(\operatorname{dim} V_{i}^{\natural}\right) q^{i}=$ $J(q)$, where $J(q)=\sum_{i \geq-1} c(i) q^{i}$ is the modular function $j(q)-744$, so that $\operatorname{dim} V_{i}^{\natural}=c(i)$ for all $i \geq-1$. We have $c(-1)=1, c(0)=0, c(1)=196884$. (For $i<-1$ we set $V_{i}^{\natural}=0$ and $c(i)=0$.) The action of $M$ preserves each $V_{i}^{\natural}$. 
The rank 2 lattice $L$ provides a $\mathbb{Z} \oplus \mathbb{Z}$-grading of the Lie algebra $\mathfrak{m}$. The group action of $M$ on the vertex operator algebra $V^{\natural}$ gives an action of $M$ on the vertex algebra $V^{\natural} \otimes V_{L}$, where $M$ acts trivially on $V_{L}$. This action induces an action of $M$ as Lie algebra automorphisms on $\mathfrak{m}$, preserving the grading. The Lie algebra $\mathfrak{m}$ has a natural nondegenerate invariant symmetric bilinear form which is also $M$-invariant. This form satisfies the condition $\left(\mathfrak{m}_{r}, \mathfrak{m}_{s}\right)=0$ unless $r+s=0$, for $r, s \in \mathbb{Z} \oplus \mathbb{Z}$. Lemma 2.2 below is one of the main theorems of [4] (cf. [19] for some clarification), and follows from the "no-ghost" theorem from string theory. Although the statement of the theorem [16] involves vector spaces over $\mathbb{R}$, after the linear isomorphism given below is established over $\mathbb{R}$ we extend the isomorphism to $\mathbb{C}$.

Lemma 2.2 As a $\mathbb{Z} \oplus \mathbb{Z}$-graded Lie algebra and $M$-module, $\mathfrak{m}$ satisfies:

$$
\mathfrak{m}=\left(\coprod_{m, n<0} \mathfrak{m}_{(m, n)}\right) \oplus \mathfrak{m}_{(-1,1)} \oplus \mathfrak{m}_{(0,0)} \oplus \mathfrak{m}_{(1,-1)} \oplus\left(\coprod_{m, n>0} \mathfrak{m}_{(m, n)}\right)
$$

where there are natural isomorphisms

$$
\begin{gathered}
\mathfrak{m}_{(m, n)} \cong V_{m n}^{\natural} \text { as an } M \text {-module for }(m, n) \neq(0,0), \\
\mathfrak{m}_{(0,0)} \cong \mathbb{C} \oplus \mathbb{C}, \text { a trivial } M \text {-module. }
\end{gathered}
$$

Furthermore, the $M$-invariant bilinear form is preserved under these isomorphisms, i.e., the pairing between $\mathfrak{m}_{(m, n)}$ and $\mathfrak{m}_{(-m,-n)}$ corresponds to the bilinear form on $V_{m n}^{\natural}$.

We define

$$
\mathfrak{n}^{+}=\coprod_{m, n>0} \mathfrak{m}_{(m, n)} \oplus \mathfrak{m}_{(1,-1)}
$$

and

$$
\mathfrak{n}^{-}=\coprod_{m, n<0} \mathfrak{m}_{(m, n)} \oplus \mathfrak{m}_{(-1,1)}
$$

so that

$$
\mathfrak{m}=\mathfrak{n}^{-} \oplus \mathfrak{m}_{(0,0)} \oplus \mathfrak{n}^{+}
$$

It follows from the definition of $\mathfrak{m}$ that

$$
\mathfrak{m}_{(-1,1)} \oplus \mathfrak{m}_{(0,0)} \oplus \mathfrak{m}_{(1,-1)} \cong \mathfrak{g l}_{2}
$$

To make this explicit, let

$$
1 \rightarrow\langle\kappa\rangle \rightarrow \hat{L} \rightarrow L \rightarrow 1
$$

be the central extension of $L$ with $\kappa$ of order 2 and commutator map given by $\kappa^{\langle\alpha, \beta\rangle}, \alpha, \beta \in L$ (cf. [12, Ch. 7]). We choose elements $a, b \in \hat{L}$ to satisfy 
$\bar{a}=(1,1), \bar{b}=(1,-1) \in L$. As in [19], appropriate elements of $V^{\natural} \otimes V_{L}$ are used to denote their equivalence classes in $\mathfrak{m}$. Let $\iota$ denote the natural map from $\hat{L}$ to $V_{L}$, so that for $c \in \hat{L}, \iota(c)$ is identified with $1 \otimes \iota(c) \in 1 \otimes \mathbb{C}\{L\} \subset S \otimes \mathbb{C}\{L\}=V_{L}$, where $S$ is the symmetric algebra of the negatively graded subspace of the appropriate Heisenberg algebra and where $\mathbb{C}\{L\}$ is the "twisted group algebra" of $L$ (cf. [12, Ch. 7]).

Consider the elements $e=1 \otimes \iota(b) \in \mathfrak{m}_{(1,-1)}, f=1 \otimes \iota\left(b^{-1}\right) \in \mathfrak{m}_{(-1,1)}, h=$ $\bar{b}(-1) \otimes \iota(1) \in \mathfrak{m}_{(0,0)}$ and $z=\bar{a}(-1) \otimes \iota(1) \in \mathfrak{m}_{(0,0)}$ in $\mathfrak{m}$. Then $e, f$ and $h$ span a copy of $\mathfrak{s l}_{2}$, with relations $[e, f]=h,[h, e]=2 e$ and $[h, f]=-2 f$. Since $z$ commutes with $e, f$ and $h$, the above elements span a copy of $\mathfrak{g l}_{2}$ in $\mathfrak{m}$. Under the adjoint action $\mathfrak{m}$ is a $\mathfrak{g l}_{2}$-module, and the element $z$ acts on $\mathfrak{m}_{(m, n)}$ as the scalar $-m-n$ and $h$ acts on $\mathfrak{m}_{(m, n)}$ as the scalar $m-n$. Note that $\mathfrak{m}_{(-1,1)} \oplus \mathfrak{m}_{(0,0)} \oplus \mathfrak{m}_{(1,-1)}$ is a trivial $M$-module. Consequently, $\mathfrak{m}$ is a $\left(\mathfrak{g l}_{2}, M\right)$ module, i.e., $\mathfrak{m}$ is both a $\mathfrak{g l}_{2}$ - and an $M$-module and these actions commute.

The Lie algebra $\mathfrak{m}$ is a generalized Kac-Moody algebra (see [4], where the definition used there allows that $\mathfrak{m}$ might be a generalized Kac-Moody algebra with some possibly nontrivial "outer" derivations adjoined, and see also [19]). In fact, $\mathfrak{m}$ is shown in [19] to be isomorphic to $\mathfrak{g}(A) / \mathfrak{c}$, where $\mathfrak{g}(A)$ is the generalized Kac-Moody algebra associated to the following matrix $A$ and $\mathfrak{c}$ is the full center of $\mathfrak{g}(A)$ :

$$
A=\left(\begin{array}{c|ccc|ccc|c}
2 & 0 & \cdots & 0 & -1 & \cdots & -1 & \cdots \\
\hline 0 & -2 & \cdots & -2 & -3 & \cdots & -3 & \\
\vdots & \vdots & \ddots & \vdots & \vdots & \ddots & \vdots & \cdots \\
0 & -2 & \cdots & -2 & -3 & \cdots & -3 & \\
\hline-1 & -3 & \cdots & -3 & -4 & \cdots & -4 & \\
\vdots & \vdots & \ddots & \vdots & \vdots & \ddots & \vdots & \cdots \\
-1 & -3 & \cdots & -3 & -4 & \cdots & -4 & \\
\hline \vdots & & \vdots & & & \vdots & &
\end{array}\right) .
$$

Consider the index set $\mathcal{I}_{0}=\{-1,1,2,3, \cdots\}$. We shall take our index set $I$, indexing the generators and simple roots of $\mathfrak{g}(A)$, to be the set $\mathcal{I}$ of pairs $(i, k) \in \mathcal{I}_{0} \oplus \mathbb{Z}_{+}$with $1 \leq k \leq c(i)$, reflecting the block form of the matrix. Thus the block in position $(i, j) \in \mathcal{I}_{0} \times \mathcal{I}_{0}$ has size $c(i) \times c(j)$, and entries $-(i+j)$ for $i, j \in \mathcal{I}_{0}$. Then $\mathfrak{g}(A)$ has generators $e_{i k}, h_{i k}, f_{i k}$ and simple roots $\alpha_{i k},(i, k) \in \mathcal{I}$. We will write $e_{-1,1}$ as $e_{-1}, f_{-1,1}$ as $f_{-1}$ and $h_{-1,1}$ as $h_{-1}$. Note that the simple roots $\alpha_{i k}$ for fixed $i \in \mathcal{I}_{0}, 1 \leq k \leq c(i)$, all have the same values on the Cartan subalgebra $\mathfrak{h} \subset \mathfrak{h}^{e}$. Under the isomorphism between $\mathfrak{g}(A) / \mathfrak{c}$ and $\mathfrak{m}$, the subalgebras $\mathfrak{n}^{ \pm} \subset \mathfrak{g}(A)$ are identified with the subalgebras $\mathfrak{n}^{ \pm} \subset \mathfrak{m}$ and the elements $h_{i k}$ for $(i, k) \in \mathcal{I}$ map to $\left(\frac{1-i}{2}\right) h+\left(\frac{1+i}{2}\right) z \in \mathfrak{m}_{(0,0)}$. The $\mathbb{Z} \oplus \mathbb{Z}$-grading of the Lie algebra $\mathfrak{m}$ given by (4) is determined by the root space grading of $\mathfrak{m}$, where the elements of $\mathfrak{m}^{\alpha_{i k}}$ are given degree $(1, i)$ for $1 \leq k \leq c(i)$. 
The Lie algebra $\mathfrak{g}(A)$ satisfies the hypothesis of Theorem 2.1. Therefore, setting

$$
\begin{aligned}
& \mathcal{V}_{i}=\coprod_{1 \leq k \leq c(i)} U\left(\mathbb{C} f_{-1}\right) \cdot f_{i k}, \\
& \mathcal{V}_{i}^{\prime}=\coprod_{1 \leq k \leq c(i)} U\left(\mathbb{C} e_{-1}\right) \cdot e_{i k}
\end{aligned}
$$

for $i>0$ and defining

$$
\mathcal{V}=\coprod_{i>0} \mathcal{V}_{i} \text { and } \mathcal{V}^{\prime}=\coprod_{i>0} \mathcal{V}_{i}^{\prime}
$$

it follows (see [19]) that

$$
\mathfrak{m}=\mathfrak{u}^{+} \oplus \mathfrak{g l}_{2} \oplus \mathfrak{u}^{-}
$$

with $\mathfrak{u}^{-}=L(\mathcal{V})$ and $\mathfrak{u}^{+}=L\left(\mathcal{V}^{\prime}\right)$. In terms of the decomposition (4),

$$
\begin{aligned}
\mathfrak{u}^{+} & =\coprod_{m, n>0} \mathfrak{m}_{(m, n)}, \\
\mathfrak{u}^{-} & =\coprod_{m, n<0} \mathfrak{m}_{(m, n)}
\end{aligned}
$$

Note that the subspaces $\mathfrak{u}^{ \pm}$are $\left(\mathfrak{g l}_{2}, M\right)$-modules.

Lemma 2.3 Let $\mathcal{V}, \mathcal{V}_{i}$ and $\mathfrak{u}^{ \pm}$be as above. The $\mathbb{Z} \oplus \mathbb{Z}$-graded vector spaces $\mathcal{V}$, $\mathcal{V}_{i}$ and $\mathfrak{u}^{ \pm}$are $\left(\mathfrak{g l}_{2}, M\right)$-modules. For $i>0$, let $W_{i}$ denote the (unique up to isomorphism) irreducible $\mathfrak{g l}_{2}$-module of dimension $i$ on which $z$ acts as $i+1$. Then $\mathcal{V}_{i} \cong W_{i} \otimes V_{i}^{\natural}$, where $W_{i}$ is regarded as a trivial $M$-module and $V_{i}^{\natural}$ as a trivial $\mathfrak{g l}_{2}$-module. Furthermore, as $M$-modules, $\mathcal{V}_{i} \cong i V_{i}^{\natural}$ and, for $m, n>0$,

$$
\left(\mathcal{V}_{i}\right)_{(-m,-n)} \cong \begin{cases}V_{i}^{\natural} & \text { if } m+n=i+1 \\ 0 & \text { otherwise. }\end{cases}
$$

Proof: Clearly, the space of highest weight vectors of $\mathcal{V}_{i}$ has basis $\left\{f_{i k} \mid 1 \leq k \leq\right.$ $c(i)\}$ and each $f_{i k}$ has degree $(-1,-i)$. Since $h$ acts on each $f_{i k}$ as multiplication by $i-1$ and $z$ acts as multiplication by $i+1$, each of these highest weight vectors generates a submodule isomorphic to $W_{i}$. Therefore,

$$
\mathcal{V}_{i} \cong W_{i} \otimes \coprod_{1 \leq k \leq c(i)} \mathbb{C} f_{i k}
$$

The isomorphism in [19] between $\mathfrak{g}(A) / \mathfrak{c}$ and $\mathfrak{m}$ is such that the subspace $\coprod_{1 \leq k \leq c(i)}(\mathfrak{g}(A) / \mathfrak{c})^{\alpha_{i k}}$ is mapped isomorphically to $\mathfrak{m}_{(1, i)}$, so the images of the $f_{i k}$ (respectively, the $e_{i k}$ ) for $1 \leq k \leq c(i)$ form a basis for the space $\mathfrak{m}_{(-1,-i)}$ 
(respectively, $\left.\mathfrak{m}_{(1, i)}\right)$. Identifying the space $\mathfrak{m}_{(-1,-i)}$ with the $M$-module $V_{i}^{\natural}$ via Lemma 2.2, we have

$$
\coprod_{1 \leq k \leq c(i)} \mathbb{C} f_{i k} \cong V_{i}^{\natural} .
$$

In this way we give the $\mathcal{V}_{i}$ (and therefore $\mathcal{V}$ ) $M$-module structures, so they are $\left(\mathfrak{g l}_{2}, M\right)$-modules.

Just as in the case of the copy of $\mathfrak{g l}_{2}=\operatorname{span}\{e, f, h, z\}$ inside $\mathfrak{m}=P_{1} / R$ described above, it is easy to identify copies of the vector spaces $\mathcal{V}$ and $\mathcal{V}^{\prime}$ inside $\mathfrak{m}$ : For $i>0, \mathfrak{m}_{(-1,-i)}$ consists of highest weight vectors for $\mathfrak{g l}_{2}$. The sum of all of the $\mathfrak{g l}_{2}$-modules generated by the $\mathfrak{m}_{(-1,-i)}, i>0$, is a copy of $\mathcal{V}$ contained in $\mathfrak{m}$. The space $\mathcal{V}^{\prime}$ can be analogously identified in $\mathfrak{m}$. We know that the Lie subalgebras of $\mathfrak{m}$ generated by $\mathcal{V}$ and $\mathcal{V}^{\prime}$ are free Lie algebras. This suggests the following:

Conjecture: The associative algebra generated by the set $\left\{v_{0} \mid v \in \mathcal{V}\right\}$ of weight-zero components of the vertex operators $Y(v, z)(v \in \mathcal{V})$ and the associative algebra generated by $\left\{v_{0} \mid v \in \mathcal{V}^{\prime}\right\}$, acting on $\mathfrak{m}$, are free associative algebras. Equivalently, these algebras are isomorphic to $T(\mathcal{V})$ and $T\left(\mathcal{V}^{\prime}\right)$, respectively.

The Lie algebra $\mathfrak{m}$ is not the only quotient of $\mathfrak{g}(A)$ which has an action of $M$. We now construct another example. Let $\mathfrak{h}_{i}, i \in \mathbb{Z}_{+}$, be the span of the $h_{i k}$ for $1 \leq k \leq c(i)$. Let $\mathfrak{c}_{i}=\mathfrak{c} \cap \mathfrak{h}_{i}$. Note that if $1 \leq k, l \leq c(i)$, then $h_{i k}-h_{i l} \in \mathfrak{c}_{i}$ (since the corresponding columns of $A$ are identical), and in fact these elements span $\mathfrak{c}_{i}$, so that $\operatorname{dim} \mathfrak{h}_{i} / \mathfrak{c}_{i}=1$. Define $\mathfrak{c}^{\prime}=\coprod_{i=1}^{\infty} \mathfrak{c}_{i}$, which is a central ideal of $\mathfrak{g}(A)$.

Proposition 2.4 The action of $M$ on the Lie algebra $\mathfrak{m}$ lifts naturally to an action of $M$ on the Lie algebra $\mathfrak{g}(A) / \mathfrak{c}^{\prime}$, in such a way that $M$ acts trivially on $\mathfrak{h} / \mathfrak{c}^{\prime}$.

Proof: We identify the elements $e_{i k}$ and $f_{i k},(i, k) \in \mathcal{I}$, in the Lie algebras $\mathfrak{g}(A)$, $\mathfrak{g}(A) / \mathfrak{c}^{\prime}$ and $\mathfrak{m}$. The invariant bilinear form on $\mathfrak{g}(A)$ is preserved, in the obvious sense, when we take quotients of $\mathfrak{g}(A)$ by central ideals. By $(11)$, for each $i>0$ we have that the span of the $f_{i k}$ and the span of the $e_{i k}, 1 \leq k \leq c(i)$, are $M$-modules. Recall that the $e_{-1}$ and $f_{-1}$ are fixed by the action of $M$. Since $h_{i k}-h_{i l} \in \mathfrak{c}_{i}$ for $1 \leq k, l \leq c(i)$, we can write the image of each $h_{i k}$ in $\mathfrak{g}(A) / \mathfrak{c}^{\prime}$ as $h_{i}$. The Lie algebra $\mathfrak{g}(A) / \mathfrak{c}^{\prime}$ then has a presentation with generators $e_{i k}, f_{i k}, h_{i}$, $i \in \mathcal{I}_{0}, 1 \leq k \leq c(i)$ and relations induced by the relations (R1)-(R5) (the relation (R6) is vacuous) of $\mathfrak{g}(A)$ :

(R1) $\left[h_{i}, h_{j}\right]=0$,

(R2) $\left[h_{l}, e_{i k}\right]=-(l+i) e_{i k}$,

(R3) $\left[h_{l}, f_{i k}\right]=(l+i) f_{i k}$, 
(R4) $\left[e_{i k}, f_{j m}\right]=\left(e_{i k}, f_{j m}\right) h_{i}$,

(R5) $\left(\operatorname{ad} e_{-1}\right)^{i} e_{i k}=0$ and $\left(\operatorname{ad} f_{-1}\right)^{i} f_{i k}=0$

for $i, j \in \mathcal{I}_{0}, 1 \leq k \leq c(i), 1 \leq m \leq c(j)$.

In $\mathfrak{g}(A) / \mathfrak{c}^{\prime}$ let $M$ act trivially on $\mathfrak{h} / \mathfrak{c}^{\prime}$, so that $M$ acts trivially on all the $h_{i}$. Relations (R1), (R2), (R3) and (R5) are $M$-invariant by inspection. The relation (R4) is equivalent to the assertion that for all $e \in \coprod_{1 \leq k \leq c(i)} \mathbb{C} e_{i k}$ and $f \in \coprod_{1 \leq k \leq c(i)} \mathbb{C} f_{i k}$

$$
[e, f]=(e, f) h_{i} .
$$

The form $(\cdot, \cdot)$ is $M$-invariant on $\coprod_{1 \leq k \leq c(i)} \mathbb{C} e_{i k} \times \coprod_{1 \leq k \leq c(i)} \mathbb{C} f_{i k}$, because by Lemma 2.2, the form is $M$-invariant on $\mathfrak{m}$. Since $\left(e_{i k}, \bar{f}_{l m}\right)=0$ if $i \neq l$, we conclude that relation (R4) is also $M$-invariant.

Of course, $M$ also acts naturally on those generalized Kac-Moody algebras formed by taking further quotients of $\mathfrak{g}(A) / \mathfrak{c}^{\prime}$ by central ideals.

\subsection{Local Lie algebras}

We now review some facts about local Lie algebras. These will be used (in Section 3) in the construction of certain generalized Kac-Moody algebras. The material in this subsection is applicable for an arbitrary field of characteristic zero.

Definition: A local Lie algebra is a graded vector space $\mathfrak{a}=\mathfrak{a}_{-1} \oplus \mathfrak{a}_{0} \oplus \mathfrak{a}_{1}$ with skew-symmetric bilinear maps $[\cdot, \cdot]_{l o c}: \mathfrak{a}_{i} \times \mathfrak{a}_{j} \rightarrow \mathfrak{a}_{i+j}$ for $|i|,|j|,|i+j| \leq 1$, satisfying:

i. $[\cdot, \cdot]]_{l o c}: \mathfrak{a}_{0} \times \mathfrak{a}_{0} \rightarrow \mathfrak{a}_{0}$ gives $\mathfrak{a}_{0}$ the structure of a Lie algebra.

ii. $[\cdot, \cdot]]_{l o c}: \mathfrak{a}_{0} \times \mathfrak{a}_{ \pm 1} \rightarrow \mathfrak{a}_{ \pm 1}$ gives $\mathfrak{a}_{ \pm 1}$ the structure of an $\mathfrak{a}_{0}$-module.

iii. $[\cdot, \cdot]$ loc $: \mathfrak{a}_{-1} \times \mathfrak{a}_{1} \rightarrow \mathfrak{a}_{0}$ gives a map $\mathfrak{a}_{-1} \otimes \mathfrak{a}_{1} \rightarrow \mathfrak{a}_{0}$ of $\mathfrak{a}_{0}$-modules.

Let $\mathfrak{l}=\coprod_{i \in \mathbb{Z}} \mathfrak{l}_{i}$ be a $\mathbb{Z}$-graded Lie algebra (with product $[\cdot, \cdot]$ ). Define $\left.[\cdot, \cdot]\right]_{l o c}$ : $\mathfrak{l}_{i} \times \mathfrak{l}_{j} \rightarrow \mathfrak{l}_{i+j}$ to be the restriction of $[\cdot, \cdot]$ to $\mathfrak{l}_{i} \times \mathfrak{l}_{j}$ whenever $|i|,|j|,|i+j| \leq 1$. Then $\mathfrak{l}_{-1} \oplus \mathfrak{l}_{0} \oplus \mathfrak{l}_{1}$ has the structure of a local Lie algebra, which we call the local part of $\mathfrak{l}$ and denote by $\mathfrak{l}_{\text {loc }}$.

We say that two local Lie algebras $\mathfrak{a}, \mathfrak{b}$ are isomorphic if there is an isomorphism $\phi: \mathfrak{a} \rightarrow \mathfrak{b}$ of graded vector spaces such that $\phi\left[a_{i}, b_{j}\right]_{l o c}=\left[\phi a_{i}, \phi b_{j}\right]_{l o c}$ for all $a_{i} \in \mathfrak{a}_{i}, b_{j} \in \mathfrak{a}_{j},|i|,|j|,|i+j| \leq 1$. A homomorphism of local Lie algebras is defined analogously.

Definition: A $\mathbb{Z}$-graded Lie algebra $\mathfrak{l}=\coprod_{i \in \mathbb{Z}} \mathfrak{l}_{i}$ is associated to the local Lie algebra $\mathfrak{a}=\mathfrak{a}_{-1} \oplus \mathfrak{a}_{0} \oplus \mathfrak{a}_{1}$ if $\mathfrak{l}_{\text {loc }}$ is isomorphic to $\mathfrak{a}$, and $\mathfrak{l}$ is generated by $\mathfrak{l}_{-1} \oplus \mathfrak{l}_{0} \oplus \mathfrak{l}_{1}$. 
The following result is due to Kac [20, Propositions 4 and 7] in case $\mathfrak{a}$ is finite-dimensional over a field of characteristic zero. The same proof remains valid for arbitrary $\mathfrak{a}$.

Proposition 2.5 Let $\mathfrak{a}=\mathfrak{a}_{-1} \oplus \mathfrak{a}_{0} \oplus \mathfrak{a}_{1}$ be a local Lie algebra. Then up to isomorphism there is a unique $\mathbb{Z}$-graded Lie algebra $\mathfrak{a}_{\max }=\coprod_{i \in \mathbb{Z}} \mathfrak{a}_{i}$ which is associated to $\mathfrak{a}$ and is maximal in the sense that any graded Lie algebra associated to $\mathfrak{a}$ is a quotient of $\mathfrak{a}_{\max }$. Furthermore, $\mathfrak{a}_{\max }^{ \pm}$is the free Lie algebra $L\left(\mathfrak{a}_{ \pm 1}\right)$ (recall the notation in Section 2.1). If $(\cdot, \cdot)_{l o c}$ is a symmetric bilinear form on $\mathfrak{a}$ which is invariant with respect to $[\cdot, \cdot \cdot]_{l o c}$, in the obvious sense, then $(\cdot, \cdot)_{\text {loc }}$ has a unique extension to an invariant symmetric bilinear form $(\cdot, \cdot)$ on $\mathfrak{a}_{\max }$.

\section{Construction of a Lie algebra from a Kac- Moody algebra and a module}

In this section we will give a construction of a Lie algebra starting from a Kac-Moody algebra and a suitable module for this algebra. We will show that every generalized Kac-Moody algebra in which no pair of distinct imaginary simple roots is orthogonal arises from our construction (so that in particular, the Monster Lie algebra does).

Let $\Gamma$ be an index set, and let $V=\coprod_{\gamma \in \Gamma} V_{\gamma}$ be a $\Gamma$-graded vector space over $\mathbb{F}$, which for the general considerations in this section, through Corollary 3.1, we take to be an arbitrary field. For a subset $S \subset \Gamma$ let

$$
V_{(S)}=\coprod_{\gamma \in \Gamma \backslash S} V_{\gamma}
$$

Clearly, $V_{(S)} \cap V_{(T)}=V_{(S \cup T)}$ and so $\left\{V_{(S)} \mid S \subset \Gamma, S\right.$ finite $\}$ forms a base of neighborhoods of 0 for a topology on $V$. As usual we give the base field the discrete topology. Let $V^{\prime} \subset V^{*}$ denote the space of continuous linear functionals on $V$. Note that the topology of $V$ and hence the space $V^{\prime}$ depend on the grading of $V$. Using a given involution of $\Gamma$, i.e., a map

$$
\begin{aligned}
& \Gamma \longrightarrow \Gamma \\
& \gamma \longmapsto \gamma^{\prime}
\end{aligned}
$$

of order one or two, we define a $\Gamma$-grading on $V^{\prime}$ by identifying $\left(V_{\gamma}\right)^{*}$ with

$$
V_{\gamma^{\prime}}^{\prime}=\left\{v^{\prime} \in V^{*} \mid v^{\prime}(v)=0 \text { for } v \in V_{(\{\gamma\})}\right\}
$$

for $\gamma \in \Gamma$; then

$$
V^{\prime}=\coprod_{\gamma \in \Gamma} V_{\gamma}^{\prime}
$$


(Later, we shall take $\Gamma$ to be an abelian group and $\gamma^{\prime}$ to be $-\gamma$.)

We say that a bilinear form $(\cdot, \cdot)$ on $V$ is compatible with the grading and with a given involution $\gamma \mapsto \gamma^{\prime}$ of $\Gamma$ if $\left(V_{\gamma}, V_{\mu}\right)=0$ if $\mu \neq \gamma^{\prime}$. We say that $V$ is finite-dimensionally graded if each $V_{\gamma}$ is finite-dimensional.

Suppose that $(\cdot, \cdot)$ is a nondegenerate (symmetric) bilinear form on $V$ which is compatible with the grading and with a given involution $\gamma \mapsto \gamma^{\prime}$. Then the restriction of $(\cdot, \cdot)$ to each $V_{\gamma} \times V_{\gamma^{\prime}}$ is nondegenerate. It follows that if $V$ is finite-dimensionally graded, then for $f \in V^{\prime}$ there exists a unique $v_{f} \in V$ such that

$$
f(v)=\left(v_{f}, v\right)
$$

for all $v \in V$.

Lemma 3.1 Let $\mathfrak{a}_{0}$ be a Lie algebra finite-dimensionally graded by $\Gamma$ (as a vector space) and let $\mathfrak{a}=\mathfrak{a}_{-1} \oplus \mathfrak{a}_{0} \oplus \mathfrak{a}_{1}$ be an $\mathfrak{a}_{0}$-module graded by $\{-1,0,1\}$. Let $(\cdot, \cdot)$ be an $\mathfrak{a}_{0}$-invariant symmetric bilinear form on $\mathfrak{a}$. Assume that $\left(\mathfrak{a}_{i}, \mathfrak{a}_{j}\right)=0$ whenever $i+j \neq 0$, that the restriction of $(\cdot, \cdot)$ to $\mathfrak{a}_{0} \times \mathfrak{a}_{0}$ is nondegenerate and compatible with the grading on $\mathfrak{a}_{0}$ and with a given involution on $\Gamma$, and that for any $a_{ \pm 1} \in \mathfrak{a}_{ \pm 1}$ the linear functional

$$
a_{0} \longmapsto\left(a_{-1}, a_{0} \cdot a_{1}\right)
$$

is continuous on $\mathfrak{a}_{0}$. Then there is a unique local Lie algebra structure on $\mathfrak{a}$ which extends the $\mathfrak{a}_{0}$-module structure of $\mathfrak{a}$ and such that $(\cdot, \cdot)$ is invariant with respect to this structure.

Proof: Let $a_{i} \in \mathfrak{a}_{i}$ for $i=-1,0,1$. We (must) define

$$
\left[a_{0}, a_{ \pm 1}\right]_{l o c}=-\left[a_{ \pm 1}, a_{0}\right]_{l o c}=a_{0} \cdot a_{ \pm 1} .
$$

The rest of $[\cdot, \cdot]_{l o c}$ is determined uniquely as follows: By hypothesis, the linear functional

$$
\begin{aligned}
& \mathfrak{a}_{0} \longrightarrow \mathbb{F} \\
& a_{0} \longmapsto\left(a_{-1},\left[a_{1}, a_{0}\right]_{l o c}\right)
\end{aligned}
$$

is continuous. Since $(\cdot, \cdot)$ is nondegenerate and compatible with the grading on $\mathfrak{a}_{0}$ there is a unique element of $\mathfrak{a}_{0}$, which we denote by $\left[a_{-1}, a_{1}\right]_{\text {loc }}$, such that

$$
\left(\left[a_{-1}, a_{1}\right]_{l o c}, a_{0}\right)=\left(a_{-1},\left[a_{1}, a_{0}\right]_{l o c}\right)
$$

for all $a_{0} \in \mathfrak{a}_{0}$. Clearly, $[\cdot, \cdot]_{l o c}$ is bilinear on $\mathfrak{a}_{-1} \times \mathfrak{a}_{1}$. Set $\left[a_{1}, a_{-1}\right]_{l o c}=$ $-\left[a_{-1}, a_{1}\right]_{l o c}$.

Then for any $a_{0}, a_{0}^{\prime} \in \mathfrak{a}_{0}$ we have

$$
\begin{aligned}
\left(\left[\left[a_{-1}, a_{1}\right]_{l o c}, a_{0}\right]_{l o c}, a_{0}^{\prime}\right) & =\left(\left[a_{-1}, a_{1}\right]_{l o c},\left[a_{0}, a_{0}^{\prime}\right]_{l o c}\right)=\left(a_{-1},\left[a_{1},\left[a_{0}, a_{0}^{\prime}\right]_{l o c}\right]_{l o c}\right) \\
& =\left(a_{-1},\left[\left[a_{1}, a_{0}\right]_{l o c}, a_{0}^{\prime}\right]_{l o c}\right)+\left(a_{-1},\left[a_{0},\left[a_{1}, a_{0}^{\prime}\right]_{l o c}\right]_{l o c}\right) \\
& =\left(\left[a_{-1},\left[a_{1}, a_{0}\right]_{l o c}\right]_{l o c}, a_{0}^{\prime}\right)+\left(\left[a_{-1}, a_{0}\right]_{l o c},\left[a_{1}, a_{0}^{\prime}\right]_{l o c}\right) \\
& =\left(\left[a_{-1},\left[a_{1}, a_{0}\right]_{l o c}\right]_{l o c}, a_{0}^{\prime}\right)+\left(\left[\left[a_{-1}, a_{0}\right]_{l o c}, a_{1}\right]_{l o c}, a_{0}^{\prime}\right) .
\end{aligned}
$$


In view of the nondegeneracy of $\left.(\cdot, \cdot)\right|_{\mathfrak{a}_{0} \times \mathfrak{a}_{0}}$ this implies

$$
\left[\left[a_{-1}, a_{1}\right]_{l o c}, a_{0}\right]_{l o c}=\left[a_{-1},\left[a_{1}, a_{0}\right]_{l o c}\right]_{l o c}+\left[\left[a_{-1}, a_{0}\right]_{l o c}, a_{1}\right]_{l o c} .
$$

This completes the proof that $[\cdot, \cdot]_{l o c}$ gives $\mathfrak{a}$ the structure of a local Lie algebra. The invariance of $(\cdot, \cdot)$ now follows from (12) together with the $\mathfrak{a}_{0}$-invariance of $(\cdot, \cdot)$, the skew symmetry of $[\cdot, \cdot]_{l o c}$ and the symmetry of $(\cdot, \cdot)$.

Now let $W=\coprod W_{i}$ be a direct sum of finite-dimensionally graded vector spaces. For each $i$, identify $W_{i}^{*}$ with $\left\{w^{*} \in W^{*} \mid w^{*}(w)=0\right.$ for $\left.w \in W_{j}, j \neq i\right\}$. Then $W_{i}^{\prime} \subset W_{i}^{*}$ (defined above) is identified with a subspace of $W^{*}$. We set

$$
W^{\prime}=\coprod W_{i}^{\prime} \subset W^{*} .
$$

For $w \in W, w^{\prime} \in W^{\prime}$ define $\left(w^{\prime}, w\right)=\left(w, w^{\prime}\right)=w^{\prime}(w)$. Also set $(W, W)=$ $\left(W^{\prime}, W^{\prime}\right)=0$. Thus $(\cdot, \cdot)$ is a nondegenerate symmetric bilinear form on $W \oplus W^{\prime}$.

Corollary 3.2 Let $\mathfrak{a}_{0}$ be a Lie algebra which is finite-dimensionally graded, as a Lie algebra, by an abelian group $\Gamma$. Assume that $\mathfrak{a}_{0}$ has a nondegenerate invariant symmetric bilinear form $(\cdot, \cdot)$ compatible with the grading and the involution $\gamma \mapsto-\gamma$ of the abelian group $\Gamma$. Let $W=\coprod W_{i}$ be a direct sum of finite-dimensionally $\Gamma$-graded $\mathfrak{a}_{0}$-modules. Then there is a unique $\mathfrak{a}_{0}$-module structure on $W^{\prime}$ such that the nondegenerate symmetric bilinear form $(\cdot, \cdot)$ on $W \oplus W^{\prime}$ defined above is $\mathfrak{a}_{0}$-invariant, and $W^{\prime}$ is $\Gamma$-graded as an $\mathfrak{a}_{0}$-module. Set $\mathfrak{a}_{1}=W, \mathfrak{a}_{-1}=W^{\prime}, \mathfrak{a}=\mathfrak{a}_{-1} \oplus \mathfrak{a}_{0} \oplus \mathfrak{a}_{1}$. Let $(\cdot, \cdot)$ be the nondegenerate $\mathfrak{a}_{0}$-invariant symmetric bilinear form on $\mathfrak{a}$ which extends the forms $(\cdot, \cdot)$ on $\mathfrak{a}_{0}$ and on $W \oplus W^{\prime}$, so that $\left(\mathfrak{a}_{i}, \mathfrak{a}_{j}\right)=0$ if $i+j \neq 0$, and $\left(a_{-1}, a_{1}\right)=a_{-1}\left(a_{1}\right)$ for $a_{ \pm 1} \in \mathfrak{a}_{ \pm 1}$. Then $\mathfrak{a}$ has a unique local Lie algebra structure extending the $\mathfrak{a}_{0}$-module structure of $\mathfrak{a}$ such that $(\cdot, \cdot)$ is invariant.

Proof: To prove the first assertion, we first note that for each $i$, the bilinear form $(\cdot, \cdot)$ on $W_{i} \oplus W_{i}^{\prime}$ is compatible with the grading and the involution $\gamma \mapsto-\gamma$. For $a_{0} \in \mathfrak{a}_{0}$ and $w_{i}^{\prime} \in W_{i}^{\prime}$, the linear functional

$$
\varphi: w_{i} \mapsto\left(w_{i}^{\prime}, a_{0} \cdot w_{i}\right)
$$

is continuous. In fact, it is sufficient to show this for $a_{0}$ in some $\left(\mathfrak{a}_{0}\right)_{\mu}$ and $w_{i}^{\prime}$ in some $\left(W_{i}^{\prime}\right)_{\nu}$, in which case $\varphi$ vanishes on $\left(W_{i}\right)_{\gamma}$ unless $\gamma+\mu+\nu=0$. Thus $\varphi$ is continuous. We (must) define $a_{0} \cdot w_{i}^{\prime} \in W_{i}^{\prime}$ so that

$$
\left(a_{0} \cdot w_{i}^{\prime}, w_{i}\right)=-\left(w_{i}^{\prime}, a_{0} \cdot w_{i}\right),
$$

and $a_{0} \cdot w_{i}^{\prime}$ is clearly bilinear in $a_{0}$ and $w_{i}^{\prime}$, and clearly defines an $\mathfrak{a}_{0}$-module action on $W^{\prime}$. It is straightforward to verify that $W^{\prime}$ is $\Gamma$-graded as an $\mathfrak{a}_{0}$-module. This completes the proof of the first assertion. 
To prove the remaining assertion, we use Lemma 3.1. To apply this, we must show that the linear functional

$$
\psi: a_{0} \longmapsto\left(a_{-1}, a_{0} \cdot a_{1}\right)
$$

is continuous for all $a_{ \pm 1} \in \mathfrak{a}_{ \pm 1}$. It is sufficient to verify this for $a_{1}$ in some $\left(W_{i}\right)_{\mu}$ and $a_{-1}$ in some $\left(W_{j}^{\prime}\right)_{\nu}$. Then $\psi$ vanishes on $\mathfrak{a}_{\gamma}$ unless $\gamma+\mu+\nu=0$ and hence $\psi$ is continuous, as required.

Now we return to the setting of Section 2.2. Let $S$ be a finite set and let $B=\left(b_{i j}\right)_{i, j \in S}$ be a matrix satisfying the conditions (C1)-(C3) and the additional condition $b_{i i}>0$ for all $i \in S$. Then $\mathfrak{g}(B)^{e}$ is the (symmetrizable) Kac-Moody algebra associated to $B$. Recall that the symmetric invariant bilinear form $(\cdot, \cdot)_{\mathfrak{g}(B)^{e}}$ on $\mathfrak{g}(B)^{e}$ is nondegenerate and satisfies the condition $\left(e_{i}, f_{j}\right)_{\mathfrak{g}(B)^{e}}=\delta_{i j}$ for all $i, j \in S$.

If $V$ is an (irreducible) integrable lowest weight $\mathfrak{g}(B)^{e}$-module (necessarily finite-dimensionally graded by its weight-space decomposition) with lowest weight $\lambda$, then $V^{\prime}$ carries the structure of an integrable highest weight module with highest weight $-\lambda$.

Let $\mathfrak{z}$ be a finite-dimensional abelian Lie algebra and let $\mathfrak{a}_{0}=\mathfrak{g}(B)^{e} \oplus \mathfrak{z}$, a direct sum of Lie algebras.

Let $W=\bigsqcup_{j \in J} W_{j}$ be a direct sum of $\mathfrak{a}_{0}$-modules, where each $W_{j}$ is an integrable lowest weight $\mathfrak{g}(B)^{e}$-module, with lowest weight $\mu_{j}$ and lowest weight vector $e_{j}$. (We assume that the two index sets $S$ and $J$ are disjoint.) Since each element of $\mathfrak{z}$ must act as a scalar on $W_{j}$, there is some $\gamma_{j} \in \mathfrak{z}^{*}$ such that $z \cdot w=\gamma_{j}(z) w$ for all $z \in \mathfrak{z}, w \in W_{j}$. Assume for convenience that

$$
\bigcap_{j \in J} \operatorname{Ker} \gamma_{j}=0 .
$$

Let $(\cdot, \cdot)_{\mathfrak{z}}$ be a nonsingular symmetric bilinear form on $\mathfrak{z}$ (which is necessarily $\mathfrak{z}$-invariant). Define $(\cdot, \cdot)$ on $\mathfrak{a}_{0}$ by $\left(g+z, g^{\prime}+z^{\prime}\right)=\left(g, g^{\prime}\right)_{\mathfrak{g}(B)^{e}}-\left(z, z^{\prime}\right)_{\mathfrak{z}}$ for $g, g^{\prime} \in \mathfrak{g}(B)^{e}, z, z^{\prime} \in \mathfrak{z}$. Note that this form is symmetric and nondegenerate.

Now $W^{\prime}=\coprod_{j \in J} W_{j}^{\prime}$ (see above) and each $W_{j}^{\prime}$ is an irreducible integrable highest weight $\mathfrak{g}(B)^{e}$-module, with highest weight $-\mu_{j}$ and a highest weight vector $f_{j}$ which we choose so as to satisfy the condition $\left(e_{j}, f_{j}\right)=1$. Clearly, $z \cdot w^{\prime}=-\gamma_{j}(z) w^{\prime}$ for all $z \in \mathfrak{z}$ and $w^{\prime} \in W_{j}^{\prime}$. Also, $\left(e_{i}, f_{j}\right)=\delta_{i j}$ for $i, j \in J$.

Set $\mathfrak{k}=\mathfrak{h}^{e} \oplus \mathfrak{z}$ and note that $(\cdot, \cdot)$ is nonsingular on $\mathfrak{k}$. We identify $\mathfrak{k}^{*}$ with $\left(\mathfrak{h}^{e}\right)^{*} \oplus \mathfrak{z}^{*}$. Let $(\cdot, \cdot)_{\mathfrak{h}^{e}}$ denote the restriction of the form $(\cdot, \cdot)_{\mathfrak{g}(B)^{e}}$ to $\mathfrak{h}^{e}$. Then for $\mu \in\left(\mathfrak{h}^{e}\right)^{*}$ there exists a unique $h_{\mu} \in \mathfrak{h}^{e}$ such that $\left(h_{\mu}, h\right)_{\mathfrak{h}^{e}}=\mu(h)$ for all $h \in \mathfrak{h}^{e}$ and for $\gamma \in \mathfrak{z}^{*}$ there exists a unique $z_{\gamma} \in \mathfrak{z}$ such that $\left(z_{\gamma}, z\right)_{\mathfrak{z}}=\gamma(z)$ for all $z \in \mathfrak{z}$. We denote $h_{\mu}+z_{\gamma}$ by $k_{\mu-\gamma} \in \mathfrak{k}$ and note that $\left(k_{\mu-\gamma}, k\right)=(\mu-\gamma)(k)$ for all $k \in \mathfrak{k}$. As usual, we may transfer the nonsingular forms $(\cdot, \cdot)_{\mathfrak{h}^{e}}$ to $\left(\mathfrak{h}^{e}\right)^{*}$ and $(\cdot, \cdot)_{\mathfrak{z}}$ to $\mathfrak{z}^{*}$ by defining $(\alpha, \beta)_{\left(\mathfrak{h}^{e}\right)^{*}}=\left(h_{\alpha}, h_{\beta}\right)_{\mathfrak{h}^{e}}$ for $\alpha, \beta \in\left(\mathfrak{h}^{e}\right)^{*}$ and $(\gamma, \sigma)_{\mathfrak{z}^{*}}=\left(z_{\gamma}, z_{\sigma}\right)_{\mathfrak{z}}$ for $\gamma, \sigma \in \mathfrak{z}^{*}$. There is a corresponding form $(\cdot, \cdot)$ on $\mathfrak{k}^{*}$ defined by $(\cdot, \cdot)_{\left(\mathfrak{h}^{e}\right)^{*}}-(\cdot, \cdot)_{\mathfrak{z}^{*}}$. 
Thus $(\nu, \tau)=\left(k_{\nu}, k_{\tau}\right)$ for $\nu, \tau \in \mathfrak{k}^{*}$. Note that

$$
\mathfrak{z}=\operatorname{span}\left\{z_{\gamma_{j}} \mid j \in J\right\} .
$$

With the $\mathfrak{a}_{0}$ given above and $\Gamma=\mathfrak{k}^{*}$, let

$$
\mathfrak{a}=W^{\prime} \oplus \mathfrak{a}_{0} \oplus W=\mathfrak{a}_{-1} \oplus \mathfrak{a}_{0} \oplus \mathfrak{a}_{1}
$$

be the local Lie algebra given by Corollary 3.2 and let $\mathfrak{a}_{\max }$ be the associated $\mathbb{Z}$-graded Lie algebra given by Proposition 2.5.

Next we give some basic bracket relations together with relevant definitions:

Proposition 3.3 The following relations hold in $\mathfrak{a}$ :

(a) If $i, j \in S$ then $\left[e_{i}, f_{j}\right]=\delta_{i j} h_{i}=\delta_{i j} h_{\alpha_{i}}$.

(b) If $i, j \in J$ then $\left[e_{i}, f_{j}\right]=\delta_{i j} k_{i}$, where

$$
k_{i}=k_{\mu_{i}+\gamma_{i}} .
$$

(c) If $i \in S$ and $j \in J$ then $\left[e_{i}, f_{j}\right]=0$ and $\left[f_{i}, e_{j}\right]=0$.

Proof: (a) is clear.

(b) For $i, j \in J,\left[e_{i}, f_{j}\right]$ is an element of $\mathfrak{a}_{0}$ which is orthogonal to the subalgebras $\mathfrak{n}^{ \pm} \subset \mathfrak{g}(B)^{e}$, so that $\left[e_{i}, f_{j}\right] \in \mathfrak{k}$. Now let $k \in \mathfrak{k}$. Then $\left(k,\left[e_{i}, f_{j}\right]\right)=$ $\left(\left[k, e_{i}\right], f_{j}\right)=\left(\left(\mu_{i}(k)+\gamma_{i}(k)\right) e_{i}, f_{j}\right)=\delta_{i j}\left(\mu_{i}(k)+\gamma_{i}(k)\right)$, so $\left[e_{i}, f_{j}\right]=\delta_{i j} k_{\mu_{j}+\gamma_{j}}$.

(c) follows from the definitions of $e_{j}$ and $f_{j}$.

Proposition 3.4 The following relations hold in $\mathfrak{a}$ :

(a) Let $i, j \in S$. Then $\left[h_{i}, e_{j}\right]=a_{i j} e_{j}$ and $\left[h_{i}, f_{j}\right]=-a_{i j} f_{j}$, where

$$
a_{i j}=b_{i j}=\left(\alpha_{i}, \alpha_{j}\right) .
$$

(b) Let $i \in S, j \in J$. Then $\left[h_{i}, e_{j}\right]=a_{i j} e_{j},\left[h_{i}, f_{j}\right]=-a_{i j} f_{j},\left[k_{j}, e_{i}\right]=a_{j i} e_{i}$, and $\left[k_{j}, f_{i}\right]=-a_{j i} f_{j}$, where

$$
a_{i j}=a_{j i}=\left(\alpha_{i}, \mu_{j}\right) .
$$

(c) Let $i, j \in J$. Then $\left[k_{i}, e_{j}\right]=a_{i j} e_{j}$ and $\left[k_{i}, f_{j}\right]=-a_{i j} f_{j}$, where

$$
a_{i j}=\left(\mu_{i}, \mu_{j}\right)_{\left(\mathfrak{h}^{e}\right)^{*}}-\left(\gamma_{i}, \gamma_{j}\right)_{\mathfrak{z}^{*}} .
$$

Proof: (a) is clear.

(b) We have

$$
\left[h_{i}, e_{j}\right]=\mu_{j}\left(h_{i}\right) e_{j}=\mu_{j}\left(h_{\alpha_{i}}\right) e_{j}=\left(\alpha_{i}, \mu_{j}\right) e_{j}
$$


and

$$
\left[k_{j}, e_{i}\right]=\left[k_{\mu_{j}+\gamma_{j}}, e_{i}\right]=\alpha_{i}\left(h_{\mu_{j}}\right) e_{i}=\left(\mu_{j}, \alpha_{i}\right) e_{i} .
$$

The other cases are similar.

(c) We have

$$
\begin{aligned}
{\left[k_{i}, e_{j}\right] } & =\left[k_{\mu_{i}+\gamma_{i}}, e_{j}\right]=\left[h_{\mu_{i}}-z_{\gamma_{i}}, e_{j}\right] \\
& =\left(\mu_{j}\left(h_{\mu_{i}}\right)-\gamma_{j}\left(z_{\gamma_{i}}\right)\right) e_{j}=\left(\left(\mu_{i}, \mu_{j}\right)_{\left(\mathfrak{h}^{e}\right)^{*}}-\left(\gamma_{i}, \gamma_{j}\right)_{\mathfrak{z}^{*}}\right) e_{j},
\end{aligned}
$$

and the remaining case is similar.

Let $A=\left(a_{i j}\right)_{i, j \in S \cup J}$, where the $a_{i j}$ are defined in the above proposition. Let $\mathfrak{d}_{B}$ be the derivation space associated with $\mathfrak{g}(B)$, i.e., $\mathfrak{g}(B)^{e}=\mathfrak{d}_{B} \ltimes \mathfrak{g}(B)$. Note that the containment $\mathfrak{d} \ltimes \mathfrak{g}(A) \subset \mathfrak{g}(A)^{e}$ can be strict. We assume that the restriction $(\cdot, \cdot)$ to $\mathfrak{g}(B)^{e}$ of the symmetric bilinear form $(\cdot, \cdot)$ on $\mathfrak{g}(A)^{e}$ has all the usual properties, in particular, that it is nondegenerate. The radical $\operatorname{Rad}(\cdot, \cdot)$ of the form $(\cdot, \cdot)$ on $\mathfrak{d}_{B} \ltimes \mathfrak{g}(A)$ is central in $\mathfrak{g}(A)^{e}$.

\section{Theorem 3.5}

(a) Assume that $a_{i j}=\left(\mu_{i}, \mu_{j}\right)_{\left(\mathfrak{h}^{e}\right)^{*}}-\left(\gamma_{i}, \gamma_{j}\right)_{\mathfrak{z}^{*}} \leq 0$ for all $i, j \in J$. Then A satisfies $(\mathrm{C} 1)-(\mathrm{C} 3)$ and the Lie algebra $\left(\mathfrak{d}_{B} \ltimes \mathfrak{g}(A)\right) / \operatorname{Rad}(\cdot, \cdot)$ is a Lie algebra associated to the local Lie algebra $\mathfrak{a}$. In particular, this Lie algebra is a quotient of $\mathfrak{a}_{\max }$.

(b) Assume that $\left(\mu_{i}, \mu_{j}\right)_{\left(\mathfrak{h}^{e}\right)^{*}}-\left(\gamma_{i}, \gamma_{j}\right)_{\mathfrak{z}^{*}} \leq 0$ for all $i, j \in J$ and that $\left(\mu_{i}, \mu_{j}\right)_{\left(\mathfrak{h}^{e}\right)^{*}}-\left(\gamma_{i}, \gamma_{j}\right)_{\mathfrak{z}^{*}}<0$ for all $i \neq j$. Then $\left(\mathfrak{d}_{B} \ltimes \mathfrak{g}(A)\right) / \operatorname{Rad}(\cdot, \cdot) \cong \mathfrak{a}_{\max }$.

Proof: We first prove (a). Since $W_{j}$ is an integrable lowest weight module with lowest weight $\mu_{j}$, the properties $(\mathrm{C} 1)-(\mathrm{C} 3)$ are clear.

We shall use the symbols $e_{i}^{A}, f_{i}^{A}$ and $h_{i}^{A}, i \in S \cup J$, to denote the generators of $\mathfrak{g}(A)$, and $\mathfrak{h}^{A}$ will denote the Cartan subalgebra of $\mathfrak{g}(A)$. Using the notation defined in (3) above, we have $\mathfrak{r}=\mathfrak{g}(B)+\mathfrak{h}^{A}$ and $\mathfrak{g}(A)=\mathfrak{u}^{+} \oplus \mathfrak{r} \oplus \mathfrak{u}^{-}$. Thus $\mathfrak{d}_{B} \ltimes \mathfrak{g}(A)=\mathfrak{u}^{+} \oplus \mathfrak{r}^{e} \oplus \mathfrak{u}^{-}$, where $\mathfrak{r}^{e}=\mathfrak{d}_{B} \ltimes \mathfrak{r}$. We give the Lie algebra $\mathfrak{d}_{B} \ltimes \mathfrak{g}(A)$ the $\mathbb{Z}$-grading determined by:

$$
\operatorname{deg} e_{j}=-\operatorname{deg} f_{j}= \begin{cases}0 & \text { if } j \in S \\ 1 & \text { if } j \in J .\end{cases}
$$

Note that $\operatorname{Rad}(\cdot, \cdot) \subset\left(\mathfrak{d}_{B} \ltimes \mathfrak{g}(A)\right)_{0}$, so that we can identify the elements $e_{i}^{A}$ and $f_{i}^{A}$ with their images in $\left(\mathfrak{d}_{B} \ltimes \mathfrak{g}(A)\right) / \operatorname{Rad}(\cdot, \cdot)$. Let $\mathfrak{l}=\left(\mathfrak{d}_{B} \ltimes \mathfrak{g}(A)\right) / \operatorname{Rad}(\cdot, \cdot)$. As $\mathfrak{d}_{B} \cap \operatorname{Rad}(\cdot, \cdot)=0$, we can also identify $\mathfrak{d}_{B}$ with its image in $\mathfrak{l}$. The $\mathbb{Z}$-grading defined on $\mathfrak{d}_{B} \ltimes \mathfrak{g}(A)$ induces a $\mathbb{Z}$-grading on $\mathfrak{l}$.

Then $\mathfrak{l}_{1}=\coprod_{j \in J} U\left(\mathfrak{n}_{S}^{+}\right) e_{j}^{A}, \mathfrak{l}_{-1}=\coprod_{j \in J} U\left(\mathfrak{n}_{S}^{-}\right) f_{j}^{A}$ and $\mathfrak{l}_{0}=\mathfrak{r}^{e} / \operatorname{Rad}(\cdot, \cdot)$. The subspace $\mathfrak{l}_{\text {loc }}=\mathfrak{l}_{-1} \oplus \mathfrak{l}_{0} \oplus \mathfrak{l}_{1}$ is the local part of the Lie algebra $\mathfrak{l}\left(\mathfrak{l}_{l o c}\right.$ generates $\mathfrak{l}$ since it contains $\mathfrak{d}_{B}$ and the generators $e_{i}^{A}$ and $\left.f_{i}^{A}, i \in S \cup J\right)$. 
Thus, by Proposition 2.5, to prove that $\mathfrak{l}$ is a quotient of $\mathfrak{a}_{\max }$ it is sufficient to prove $\mathfrak{l}_{l o c} \cong \mathfrak{a}$. By the uniqueness assertion in Lemma 3.1, it is enough to give a linear isomorphism

$$
\varphi: \mathfrak{l}_{l o c} \rightarrow \mathfrak{a}
$$

preserving the gradings by the set $\{-1,0,1\}$, such that for $x \in \mathfrak{l}_{0}, y \in \mathfrak{l}_{\text {loc }}$,

$$
[\varphi(x), \varphi(y)]=\varphi([x, y])
$$

and such that for all $x, y \in \mathfrak{l}_{l o c}$,

$$
(\varphi(x), \varphi(y))=(x, y) .
$$

To do this we will define a surjective homomorphism of graded vector spaces, which we still call $\varphi$,

$$
\varphi: \mathfrak{l}_{-1} \oplus \mathfrak{r}^{e} \oplus \mathfrak{l}_{1} \rightarrow \mathfrak{a}_{-1} \oplus \mathfrak{a}_{0} \oplus \mathfrak{a}_{1},
$$

and show that (13) and (14) are satisfied and that the kernel of $\varphi$ is $\operatorname{Rad}(\cdot, \cdot)$.

We define $\varphi$ as a linear map by prescribing $\varphi$ on the subspaces $\mathfrak{l}_{ \pm 1}, \mathfrak{g}(B)^{e}$ and the span of the $h_{j}^{A}$ for $j \in J$.

Each $W_{j}$ for $j \in J$ is an integrable lowest weight $\mathfrak{g}(B)^{e}$-module of weight $\mu_{j}$ and so is isomorphic to the integrable lowest weight $\mathfrak{g}(B)^{e}$-module $U\left(\mathfrak{n}_{S}^{+}\right) e_{j}^{A}$ of weight $\mu_{j}$ by an isomorphism $\varphi$ which takes the lowest weight vector $e_{j}^{A}$ to $e_{j}$. This gives us $\varphi: \mathfrak{l}_{1} \stackrel{\sim}{\rightarrow} \mathfrak{a}_{1}$. The isomorphism $\varphi: \mathfrak{l}_{-1} \stackrel{\sim}{\rightarrow} \mathfrak{a}_{-1}$ is defined analogously.

We define $\varphi$ to be the identity on $\mathfrak{g}(B)^{e}$.

To define $\varphi$ on all of $\mathfrak{r}^{e}$, all that remains is to define $\varphi$ on $h_{j}^{A} \in \mathfrak{h}^{A}$ with $j \in J$. Set $\varphi\left(h_{j}^{A}\right)=k_{j}=k_{\mu_{j}+\gamma_{j}} \in \mathfrak{k}$.

This defines a homomorphism of graded vector spaces $\varphi: \mathfrak{l}_{-1} \oplus \mathfrak{r}^{e} \oplus \mathfrak{l}_{1} \rightarrow \mathfrak{a}$. That $\varphi$ satisfies (13) follows from Proposition 3.4. This map is surjective because the $z_{\gamma_{j}} \operatorname{span} \mathfrak{z}$.

It is clear from the definition of the forms that $(x, y)=(\varphi(x), \varphi(y))$ for $x, y \in \mathfrak{g}(B)^{e}$. The invariant bilinear forms on $\mathfrak{l}_{-1} \oplus \mathfrak{r}^{e} \oplus \mathfrak{l}_{1}$ and on $\mathfrak{a}$ satisfy the relations

$$
\left(e_{i}^{A}, f_{j}^{A}\right)=\left(e_{i}, f_{j}\right)=\delta_{i j}
$$

for all $i, j \in J$. Since an invariant pairing between a lowest and highest weight $\mathfrak{g}(B)^{e}$-module is determined by its value on the pair consisting of the lowest and highest weight vectors, we conclude that $(x, y)=(\varphi(x), \varphi(y))$ for $x \in \mathfrak{l}_{1}$ and $y \in \mathfrak{l}_{-1}$. Furthermore, for $i \in S \cup J, j \in J$

$$
\begin{aligned}
\left(h_{i}^{A}, h_{j}^{A}\right)=a_{i j} & = \begin{cases}\left(\alpha_{i}, \mu_{j}+\gamma_{j}\right) & \text { if } i \in S \\
\left(\mu_{i}+\gamma_{i}, \mu_{j}+\gamma_{j}\right) & \text { if } i \in J\end{cases} \\
& = \begin{cases}\left(h_{\alpha_{i}}, k_{j}\right) & \text { if } i \in S \\
\left(k_{i}, k_{j}\right) & \text { if } i \in J\end{cases} \\
& =\left(\varphi\left(h_{i}\right), \varphi\left(h_{j}\right)\right) .
\end{aligned}
$$


Also, it is clear that for $d \in \mathfrak{d}_{B}$ and $j \in J,\left(d, h_{j}^{A}\right)=\left(\varphi(d), \varphi\left(h_{j}^{A}\right)\right)$. It follows that for all $x, y \in \mathfrak{l}_{-1} \oplus \mathfrak{r}^{e} \oplus \mathfrak{l}_{1}$, we have $(x, y)=(\varphi(x), \varphi(y))$.

The kernel of $\varphi$ clearly lies in $\mathfrak{d}_{B} \oplus \mathfrak{h}^{A}$. Let $h$ be an element of the kernel. Then for all $x \in \mathfrak{l}_{-1} \oplus \mathfrak{r}^{e} \oplus \mathfrak{l}_{1},(h, x)=(\varphi(h), \varphi(x))=0$, so that $h \in \operatorname{Rad}(\cdot, \cdot)$. Conversely, if $y \in \operatorname{Rad}(\cdot, \cdot)$, then $(\varphi(y), a)=0$ for all $a \in \mathfrak{a}$, since $\varphi$ is surjective. As the bilinear form is nondegenerate on $\mathfrak{a}_{0}$, this means that $\varphi(y)=0$. We conclude that the kernel of the map $\varphi$ is $\operatorname{Rad}(\cdot, \cdot)$, completing the proof of (a).

Part (b) then follows by Theorem 2.1 and the definition of $\mathfrak{d}_{B} \ltimes \mathfrak{g}(A)$.

Remark 3.6 After possible reordering of rows and columns, every finite rank matrix satisfying $(\mathrm{C} 1)-(\mathrm{C} 3)$ and having only finitely many positive diagonal entries arises as the matrix $A$ for a suitable choice of the generalized Cartan matrix $B$, the module $W$ and the elements $\gamma_{i}$. In fact, let $P=\left(p_{k l}\right)_{k, l \in K}$ be any matrix as indicated. Let $S=\left\{i \in K \mid p_{i i}>0\right\}$ and $J=\left\{j \in K \mid p_{j j} \leq 0\right\}$, so that $S$ is a finite set. Reorder the rows and columns of $P$ so that the rows and columns indexed by elements of $S$ precede those indexed by elements of $J$. Let $B=\left(p_{k l}\right)_{k, l \in S}$. Consider the Kac-Moody algebra $\mathfrak{g}(B)^{e}$. For $j \in J$, define $\mu_{j}$ to be any element of $\left(\mathfrak{h}^{e}\right)^{*}$ such that $\mu_{j}\left(h_{i}\right)=p_{i j}$ for $i \in S$. Let $W_{j}$ be the integrable lowest weight $\mathfrak{g}(B)^{e}$-module with lowest weight $\mu_{j}$. Define $q_{i j}=\left(\mu_{i}, \mu_{j}\right)-p_{i j}$ for $i, j \in J$ and let $Q=\left(q_{i j}\right)_{i, j \in J}$. Let $\mathfrak{z}$ be an abelian Lie algebra of (finite) dimension equal to the rank of $Q$, equipped with a nonsingular symmetric bilinear form $(\cdot, \cdot)_{\mathfrak{z}}$. Let $(\cdot, \cdot)_{\mathfrak{z}^{*}}$ be the corresponding form on $\mathfrak{z}^{*}$. Let $\mathfrak{t}$ be a vector space over $\mathbb{C}$ with basis $\left\{\eta_{j} \mid j \in J\right\}$. Define an inner product on $\mathfrak{t}$ by $\left(\eta_{i}, \eta_{j}\right)=q_{i j}$ for all $i, j \in J$. Then $\mathfrak{t} / \operatorname{Rad}(\cdot, \cdot)$ has dimension equal to rank $Q$, hence to $\operatorname{dim} \mathfrak{z}^{*}$. We may therefore identify $\mathfrak{t} / \operatorname{Rad}(\cdot, \cdot)$ with $\mathfrak{z}^{*}$. Let $\gamma_{j}$ denote the image of $\eta_{j}$ under this identification, so that $\left(\gamma_{i}, \gamma_{j}\right)_{\mathfrak{z}^{*}}=q_{i j}$ for all $i, j \in J$. For these choices of $B, W=\bigsqcup_{j \in J} W_{j}$ and the $\gamma_{j}$, the resulting matrix $A$ is equal to $P$.

We give such a construction of the Monster Lie algebra explicitly: In this case (using the notation of Section 2.3), $K=\mathcal{I}, S=\{(-1,1)\}, J=\{(i, k) \mid i \in$ $\left.\mathbb{Z}_{+}, 1 \leq k \leq c(i)\right\}$ and $B=(2)$. Thus $\mathfrak{g}(B)=\mathfrak{g}(B)^{e}=\mathfrak{s l}_{2}$, so that $\mathfrak{d}_{B}=0$ and $\operatorname{dim} \mathfrak{h}^{e}=1$. As before, we write $h_{-1}$ for $h_{-1,1}$. Since for $i \in \mathbb{Z}_{+} \mu_{(i, k)}$ depends only on $i$, we may denote it $\mu_{i}$. Then $\mu_{i}\left(h_{-1}\right)=-(i-1)$ and $\left(\mu_{i}, \mu_{j}\right)=(i-1)(j-$ $1) / 2$. Hence the entries in the $(i, j)$-block of $Q$ have value $(i+1)(j+1) / 2$. We may therefore take $\mathfrak{z}$ to be one-dimensional. If $z$ spans $\mathfrak{z}$ and satisfies $(z, z)_{\mathfrak{z}}=1$, then we have $\gamma_{i}(z)=(i+1) / \sqrt{2}$. Note that $\mathfrak{a}_{0}=\mathfrak{s l}_{2} \oplus \mathfrak{z} \cong \mathfrak{g l}_{2}$. Because the center $\mathfrak{c}$ is equal to $\operatorname{Rad}(\cdot, \cdot)$, we conclude that $\mathfrak{m}=\mathfrak{a}_{\max }$ for $\mathfrak{a}=\mathcal{V}^{\prime} \oplus \mathfrak{g l}_{2} \oplus \mathcal{V}$.

\section{Generalized Verma modules and standard mod- ules}

Given a generalized Kac-Moody Lie algebra, we observe that certain standard modules are equal to induced modules. For Lie algebras $\mathfrak{g}$ satisfying the condi- 
tions of Theorem 2.1, this means that the tensor algebra over a certain vector space has the structure of an irreducible module for $\mathfrak{g}$. In this way we show that $\mathfrak{m}$ (and $\mathfrak{g}(A) / \mathfrak{c}^{\prime}$ ) can be realized naturally as an explicitly prescribed $M$ covariant Lie algebra of operators on $T(\mathcal{V})$ (which has the form of an induced $\mathfrak{m}$ - or $\mathfrak{g}(A) / \mathfrak{c}^{\prime}$-module for certain weights $\lambda$ ) for our $M$-module $\mathcal{V}$.

\subsection{Examples of standard modules}

The conditions in the definition below of standard module appear in [2]; the definition was made in [18]. Standard modules are the only modules for which a character formula is proven ([2]; see [17], [18] and [21]). Note that standard modules are not the same as "integrable highest weight modules" for generalized Kac-Moody algebras (see [21] for the definition of "integrable" in the context of generalized Kac-Moody algebras).

Let $X$ be a $\mathfrak{g}^{e}$-module. A weight $\lambda \in\left(\mathfrak{h}^{e}\right)^{*}$ is dominant if $\left(\lambda, \alpha_{i}\right) \in \mathbb{R}$ and $\left(\lambda, \alpha_{i}\right) \geq 0$ for all $i \in I$. A weight $\lambda$ is called integral if $\frac{2 \lambda\left(h_{i}\right)}{a_{i i}} \in \mathbb{Z}$ for all $i \in I$ such that $a_{i i}>0$. Denote by $P_{+}$the set of dominant integral weights.

As in [18], define $X$ to be a standard module if $X$ is a highest weight module ([2] and [18] actually use lowest weight modules) with highest weight $\mu \in P_{+}$ and highest weight vector $x$ such that:

1. for $i \in I$, if $\left(\mu, \alpha_{i}\right)=0$ then $f_{i} \cdot x=0$;

2. if $\alpha_{i}(i \in I)$ is real then $f_{i}^{n_{i}+1} \cdot x=0$, where $n_{i}=2\left(\mu, \alpha_{i}\right) /\left(\alpha_{i}, \alpha_{i}\right)$ (necessarily a nonnegative integer).

Proposition 4.1 All standard modules are irreducible.

Proof: This follows from the character formula for a standard module stated in $[2]$ and [21]. For two different complete proofs of the character formula see [17] and $[18]$.

Let $\lambda \in P_{+}$, and consider the standard (irreducible) highest weight $\mathfrak{r}^{e}$-module $L(\lambda)$ associated to $\lambda$; it is irreducible as a $\mathfrak{g}_{S}^{e}$-module (cf. [21]). The highest weight space of $L(\lambda)$ (as a $\mathfrak{g}_{S}^{e}$-module) is a weight space for $\mathfrak{h}^{e}$, with weight $\lambda$. Let $\mathfrak{u}^{+}$act trivially on $L(\lambda)$; this gives $L(\lambda)$ the structure of an irreducible $\mathfrak{p}^{e}$-module. Define the generalized Verma module $V^{L(\lambda)}$ (cf. [25]) to be the induced module $U\left(\mathfrak{g}^{e}\right) \otimes_{U\left(\mathfrak{p}^{e}\right)} L(\lambda)$. It is clear that $V^{L(\lambda)} \cong U\left(\mathfrak{u}^{-}\right) \otimes L(\lambda)$ as vector spaces.

We make the following observation:

Proposition 4.2 Let $\lambda \in P_{+}$be an element satisfying $\left(\lambda, \alpha_{i}\right)>0$ for every imaginary simple root $\alpha_{i}(i \in I \backslash S)$. Then the generalized Verma module $V^{L(\lambda)}$ is a standard, and therefore irreducible, module for $\mathfrak{g}(A)^{e}$. 
Proof: The module $V^{L(\lambda)}$ is a highest weight module with highest weight $\lambda$, and clearly satisfies conditions 1 and 2 above.

Proposition 4.2 immediately gives a class of examples of standard modules for arbitrary generalized Kac-Moody algebras. In the case of generalized KacMoody algebras satisfying the hypothesis of Theorem 2.1, like $\mathfrak{m}$, these generalized Verma modules are particularly easy to describe. As another example, we show that the irreducible module constructed in [15] for a generalized KacMoody Lie algebra with one imaginary simple root is an example of such a generalized Verma module.

Theorem 4.3 Let $\mathfrak{g}(A)=\mathfrak{g}$ be a generalized Kac-Moody algebra such that if $\alpha_{i}$ and $\alpha_{j}$ are two distinct imaginary simple roots then $\left(\alpha_{i}, \alpha_{j}\right)=a_{i j}<0$. Let $W$ denote the direct sum of the integrable highest weight $\mathfrak{g}_{S}^{e}$-modules $U\left(\mathfrak{n}_{S}^{-}\right) \cdot f_{j}$ for all $j \in I \backslash S$. Given $\lambda \in P_{+}$such that $\left(\lambda, \alpha_{i}\right)>0$ for every imaginary simple root $\alpha_{i}(i \in I \backslash S)$, the generalized Verma module $V^{L(\lambda)}$ has the form $T(W) \otimes L(\lambda)$, and is a standard (and in particular irreducible) $\mathfrak{g}^{e}$-module.

Proof: Let $\lambda \in\left(\mathfrak{h}^{e}\right)^{*}$ be such that $\left(\lambda, \alpha_{i}\right)=\lambda\left(h_{i}\right) \neq 0$ for all $i \in S$. The generalized Verma module $V^{L(\lambda)}=U\left(\mathfrak{u}^{-}\right) \otimes L(\lambda)$ is a standard module by Proposition 4.2. Since $\mathfrak{u}^{-}$is the free Lie algebra on $W=\bigsqcup_{k} U\left(\mathfrak{n}_{S}^{-}\right) \cdot f_{k}$ (by Theorem 2.1), we conclude that $U\left(\mathfrak{u}^{-}\right) \otimes L(\lambda) \cong T(W) \otimes L(\lambda)$.

Corollary 4.4 In the setting of Theorem 4.3 assume that $\lambda\left(h_{i}\right)=0$ for all $i \in$ $S$. Then tensor algebra $T(W)$ carries the natural structure of a standard $\mathfrak{g}^{e}$ module.

Proof: The $\mathfrak{g}_{S}$-module $L(\lambda)$ is one-dimensional.

Note that Corollary 4.4 includes the case in [15], where $\mathfrak{g}_{S}$ is a Kac-Moody algebra and the generalized Kac-Moody algebra $\mathfrak{g}$ is formed by adjoining one additional imaginary simple root $\alpha_{0}$ to $\mathfrak{g}_{S}$. This is done (see [15]) by taking $\mathfrak{g}$ to be the Lie algebra associated to the matrix:

$$
\left(\begin{array}{c|c}
\left(a_{i j}\right)_{i, j \in S} & \left(a_{0 j}\right)_{j \in S} \\
\hline\left(a_{j 0}\right)_{j \in S} & a_{00}
\end{array}\right)
$$

where $a_{00} \leq 0$. The hypothesis of Theorem 4.3 is then satisfied, because the condition on the imaginary simple roots becomes vacuous. Let $f$ be the generator in $\mathfrak{n}^{-}$associated to the one imaginary simple root. Thus if $W$ denotes the integrable highest weight module $U\left(\mathfrak{n}_{S}^{-}\right) \cdot f$, then $T(W)$ is a standard module. It is isomorphic to the generalized Verma module associated to the module $\mathbb{C}_{\lambda}$ where $\left(\lambda, \alpha_{0}\right)=1$ and $(\lambda, \alpha)=0$ for simple $\alpha \in \Delta_{R}$.

Recall the matrix $A$ given in Section 2.3, so that the Lie algebra $\mathfrak{g}(A)$ has only only one real simple root and all of the $a_{i j}=\left(\alpha_{i}, \alpha_{j}\right)<0$ for $\alpha_{i}, \alpha_{j}$ imaginary simple, so the conditions of Corollary 4.4 are satisfied. In the case of 
the Lie algebra $\mathfrak{g}(A) / \mathfrak{c}^{\prime}$ of Proposition 2.4 and the Monster Lie algebra $\mathfrak{m}$, we can obtain standard modules by applying Corollary 4.4 to $\mathfrak{g}(A)$. If we choose $\lambda \in P_{+}$satisfying the conditions

$$
\begin{aligned}
& \lambda\left(h_{-1}\right)=0 \\
& \lambda\left(h_{i k}\right)=\lambda\left(h_{i j}\right) \quad \text { for each } i>0,1 \leq k, j \leq c(i),
\end{aligned}
$$

we get an irreducible generalized Verma module $V^{L(\lambda)}$ for $\mathfrak{g}(A) / \mathfrak{c}^{\prime}$. To obtain $\mathfrak{m}$-modules, given any real numbers $a, b$ such that $a>0$ and $a+b>0$ let $\lambda \in\left(h^{e}\right)^{*}$ be an element satisfying the conditions

$$
\begin{aligned}
& \lambda\left(h_{-1}\right)=0 \\
& \lambda\left(h_{i k}\right)=\lambda\left(h_{i j}\right)=a i+b \quad \text { for each } i>0,1 \leq k, j \leq c(i) .
\end{aligned}
$$

Then $\lambda \in P_{+}$, and the full center $\mathfrak{c}$ of $\mathfrak{g}(A)$ acts as zero on the module $V^{L(\lambda)}$. Thus $V^{L(\lambda)}$ is also an $\mathfrak{m}$-module.

Recall the $M$-module $\mathcal{V}$ given by (7), so that $\mathfrak{u}^{-}=L(\mathcal{V})$. We have shown that for the choices of $\lambda$ given above the $M$-module $T(\mathcal{V})=U\left(\mathfrak{u}^{-}\right)$has a natural standard module structure for $\mathfrak{g}(A) / \mathfrak{c}^{\prime}$ or for $\mathfrak{m}$.

Summarizing the above considerations, we describe $\mathfrak{m}=L(\mathcal{V}) \oplus \mathfrak{g l}_{2} \oplus L\left(\mathcal{V}^{\prime}\right)$ (and $\mathfrak{g}(A) / \mathfrak{c}^{\prime}$ ) in the following (obvious) way:

Theorem 4.5 Let $\mathcal{V}$ be given by (7). The Lie algebra $\mathfrak{m}$ can be realized in a natural way as a Lie algebra of operators on the irreducible $\mathfrak{m}$-module $T(\mathcal{V})$, identified as above with a generalized Verma module, for any weight $\lambda$ satisfying (16). The $M$-module structure on the tensor algebra $T(\mathcal{V})$ induced by the $M$ module structure on $\mathcal{V}$ is compatible with the action of $M$ on $\mathfrak{m}$. The following operators generate $\mathfrak{m}: \mathcal{V}$ acting on $T(\mathcal{V})$ by left multiplication, $\mathfrak{g l}_{2}$ acting on $T(\mathcal{V})$ via the action induced by the $\mathfrak{g l}_{2}$-module structure of $\mathcal{V}$, and $\mathcal{V}^{\prime}$ acting on $T(\mathcal{V})$ via its natural action on the induced module. Furthermore, these comments extend to the Lie algebra $\mathfrak{g}(A) / \mathfrak{c}^{\prime}$ for weights $\lambda$ satisfying (15).

We suggest that these irreducible $\mathrm{m}$-modules $T(\mathcal{V})$ (and similarly, $T\left(\mathcal{V}^{\prime}\right)$ ) are realizable in a conceptual way as the particular free associative algebras of operators described in our conjecture in Section 2.

\section{Application to Borcherds' proof of the moon- shine conjectures}

We will apply the decomposition of $\mathfrak{m}$ as $\mathfrak{u}^{+} \oplus \mathfrak{g l} \mathfrak{l}_{2} \oplus \mathfrak{u}^{-}$, where $\mathfrak{u}^{+}$and $\mathfrak{u}^{-}$are free Lie algebras, to simplify part of the proof appearing in [4] of the Conway-Norton conjectures [8]. It has already been seen [19] that the denominator identity for $\mathfrak{m}$ can be obtained with the help of the fact that $\mathfrak{u}^{-}$is a free Lie algebra (but still using Borcherds' product formula for $j(q))$. This provides one simplification of 
Borcherds' proof, because it requires less of the theory of Kac-Moody algebras to be generalized. Another simplification will be obtained here by computing the homology $H\left(\mathfrak{u}^{-}, \mathbb{C}\right)$ of the subalgebra $\mathfrak{u}^{-}$, rather than computing the homology of the Lie algebra $\mathfrak{n}^{+}$(or equivalently $\mathfrak{n}^{-}$) as in [4]. This avoids using results of [14] extended to generalized Kac-Moody Lie algebras.

We show that the replication formulas of [8] can be obtained from this homology result and calculations similar to those appearing in [6] for computing the dimensions of homogeneous subspaces of free Lie algebras.

\subsection{Homology}

It is easy to compute the homology of the free Lie algebra $L(V)$ for a vector space $V$ : The following exact sequence is a $U(L(V))=T(V)$-free resolution of the trivial module:

$$
0 \rightarrow T(V) \otimes V \stackrel{\mu}{\rightarrow} T(V) \stackrel{\epsilon}{\rightarrow} \mathbb{C} \rightarrow 0
$$

where $\mu$ is the multiplication map and $\epsilon$ is the augmentation map. Note that if a group or a Lie algebra acts on $V$ then the group or Lie algebra also acts on every term in the resolution (17) in a natural way, and therefore acts on $H(L(V), \mathbb{C})$.

One immediately observes from the resolution (17):

$$
\begin{gathered}
H_{0}(L(V), \mathbb{C})=\mathbb{C} \\
H_{1}(L(V), \mathbb{C})=V \cong L(V) /[L(V), L(V)] \\
H_{n}(L(V), \mathbb{C})=0 \text { for } n \geq 2 .
\end{gathered}
$$

(It is also well known how to compute $H_{0}(\mathfrak{a}, \mathbb{C})$ and $H_{1}(\mathfrak{a}, \mathbb{C})$ for an arbitrary Lie algebra $\mathfrak{a}[7]$.)

\subsection{Adams operations}

In this section $p, q$ and $t$ are commuting formal variables. The variables $p^{-1}$ and $q^{-1}$ will be used to index the $\mathbb{Z} \oplus \mathbb{Z}$-grading of our vector spaces. All of the $M$-modules we encounter are finite-dimensionally $\mathbb{Z} \oplus \mathbb{Z}$-graded with grading suitably truncated and will be identified with formal series in $R(M)[[p, q]]$. Definitions and results from [22] about the $\lambda$-ring $R(M)$ of finite-dimensional representations of $M$ are applicable to formal series in $R(M)[[p, q]]$. We summarize the results of [1], [22] that we use below.

Let $G$ be any finite group. The representation ring $R(G)$ is a $\lambda$-ring [22] with the $\lambda$ operation given by exterior powers, so $\lambda^{i} V=\bigwedge^{i} V$ for $V \in R(G)$.

In the following discussion we let $W, V \in R(G)$. The operation $\bigwedge^{i}$ satisfies

$$
\bigwedge^{i}(W \oplus V)=\sum_{n=0}^{i} \bigwedge^{n}(W) \otimes \bigwedge^{i-n}(V) .
$$


Define

$$
\bigwedge_{t}(W)=\bigwedge^{0}(W)+\bigwedge^{1}(W) t+\bigwedge^{2}(W) t^{2}+\cdots
$$

Then

$$
\bigwedge_{t}(V \oplus W)=\bigwedge_{t}(V) \cdot \bigwedge_{t}(W) .
$$

The Adams operations $\Psi^{k}: R(G) \rightarrow R(G)$ are defined for $W \in R(G)$ by:

$$
\frac{d}{d t} \log \bigwedge_{t}(W)=\sum_{n \geq 0}(-1)^{n} \Psi^{n+1}(W) t^{n} .
$$

We use the following properties of the $\Psi^{k}$ from [22]:

$$
\begin{aligned}
& \Psi^{k}(V \otimes W)=\Psi^{k}(V) \otimes \Psi^{k}(W) \\
& \Psi^{k}(V \oplus W)=\Psi^{k}(V) \oplus \Psi^{k}(W) .
\end{aligned}
$$

Thus the $\Psi^{k}$ are ring homomorphisms. Also

$$
\Psi^{k} \Psi^{l}(W)=\Psi^{k l}(W)
$$

For a class function $f: G \rightarrow \mathbb{C}$, define

$$
\left(\Psi^{k} f\right)(g)=f\left(g^{k}\right) .
$$

for all $g \in G$. Then if $\chi_{V}$ is a character of $G$,

$$
\chi_{\Psi^{k}(V)}(g)=\left(\Psi^{k} \chi_{V}\right)(g)
$$

(cf. $[22])$.

Now let $W$ be a finite-dimensionally $\mathbb{Z} \oplus \mathbb{Z}$-graded representation of $G$ such that $W_{\left(\gamma_{1}, \gamma_{2}\right)}=0$ for $\gamma_{1}, \gamma_{2}>0$. We shall write

$$
W=\sum_{\left(\gamma_{1}, \gamma_{2}\right) \in \mathbb{N}^{2}} W_{\left(-\gamma_{1},-\gamma_{2}\right)} p^{\gamma_{1}} q^{\gamma_{2}}
$$

identifying the graded space and formal series. We extend the definition of $\Psi^{k}$ to formal series $W \in R(G)[[p, q]]$ by defining $\Psi^{k}(p)=p^{k}, \Psi^{k}(q)=q^{k}$ and in general,

$$
\Psi^{k}\left(\sum_{\left(\gamma_{1}, \gamma_{2}\right) \in \mathbb{N}^{2}} W_{\left(-\gamma_{1},-\gamma_{2}\right)} p^{\gamma_{1}} q^{\gamma_{2}}\right)=\sum_{\left(\gamma_{1}, \gamma_{2}\right) \in \mathbb{N}^{2}} \Psi^{k}\left(W_{\left(-\gamma_{1},-\gamma_{2}\right)}\right) p^{k \gamma_{1}} q^{k \gamma_{2}} .
$$

Expressions involving $\bigwedge^{i}(W)$ for $W \in R(G)[[p, q]]$ are interpreted in the obvious way, so that (20) is meaningful and (19) and (21) are valid for our finite-dimensionally graded spaces. The identity (22) remains valid for $W \in$ $R(G)[[p, q]]$ and the $\Psi^{k}$ defined above by (29). Also, (23) - (25) remain valid. 


\subsection{Möbius inversion}

Let $G$ be a finite group and let $W$ be a finite-dimensionally $\Gamma$-graded representation of $G$, where $\Gamma$ is a finitely generated free abelian group. Then $W=\coprod_{\gamma \in \Gamma} W_{\gamma}$ where each $W_{\gamma}$ is a finite-dimensional module for $G$. We prove a Möbius inversion formula involving functions $s, t: G \times \Gamma \rightarrow \mathbb{C}$ such that for fixed $\gamma \in \Gamma, s$ and $t$ are class functions of $G$. For each $g \in G, s$ and $t$ are functions taking $\Gamma$ to $\mathbb{C}$; we sometimes write $s(\gamma), t(\gamma), \gamma \in \Gamma$. For example, we may take $t(g, \gamma)=\operatorname{Tr}\left(g \mid W_{\gamma}\right), g \in G, \gamma \in \Gamma$. If $\Gamma$ has base $\left\{\gamma_{1}, \ldots, \gamma_{k}\right\}$, and if $\nu=a_{1} \gamma_{1}+\cdots+a_{k} \gamma_{k} \in \Gamma\left(a_{i} \in \mathbb{Z}\right)$ and $d \in \mathbb{Z}$ then we say $d \mid \nu$ if $d \mid a_{i}$ for each $i$. For $\nu, \kappa \in \Gamma$ such that $\nu=d \kappa$ we write $\frac{\nu}{\kappa}=d$. Let $\mu$ be the Möbius function.

Lemma 5.1 Let $s$ and $t$ be as above and let $\nu \in \Gamma, d \in \mathbb{Z}_{+}$. Then

$$
\sum_{d \mid \nu} \Psi^{d} t\left(\frac{\nu}{d}\right)=s(\nu)
$$

if and only if

$$
t(\nu)=\sum_{d \mid \nu} \mu(d) \Psi^{d} s\left(\frac{\nu}{d}\right) .
$$

Proof: We use a standard argument (cf. [6]). Assume equation (30). Then

$$
\begin{aligned}
\sum_{d \mid \nu} \mu(d) & \Psi^{d} s\left(\frac{\nu}{d}\right)=\sum_{d \mid \nu} \mu(d) \Psi^{d}\left(\sum_{\delta \mid \frac{\nu}{d}} \Psi^{\delta} t\left(\frac{\nu}{d \delta}\right)\right) \\
& =\sum_{d \mid \nu} \mu(d) \sum_{\delta \mid \frac{\nu}{d}} \Psi^{d \delta} t\left(\frac{\nu}{d \delta}\right) \\
& =\sum_{d \delta \mid \nu} \mu(d) \Psi^{d \delta} t\left(\frac{\nu}{d \delta}\right) \\
& =\sum_{\kappa \mid \nu} \sum_{d \mid \frac{\nu}{\kappa}} \mu(d) \Psi^{\nu / \kappa} t(\kappa) \\
& =\sum_{\kappa \mid \nu} \Psi^{\nu / \kappa} t(\kappa) \sum_{d \mid \frac{\nu}{\kappa}} \mu(d)=t(\nu) .
\end{aligned}
$$

That (31) implies (30) is similar.

We will apply this inversion formula to the situation where $\Gamma=\mathbb{Z} \oplus \mathbb{Z}$ and $W \in R(G)[[p, q]]$ as in $(28)$. For a pair $(i, j) \in \mathbb{Z} \oplus \mathbb{Z}$ our definition specializes to: $k \mid(i, j)$ if $k(m, n)=(i, j)$ for some $(m, n) \in \mathbb{Z} \oplus \mathbb{Z}$. For $(i, j) \in \mathbb{Z}_{+} \oplus \mathbb{Z}_{+}$we define

$$
P(i, j)=\left\{a=\left(a_{r s}\right)_{r, s \in \mathbb{Z}_{+}} \mid a_{r s} \in \mathbb{N}, \sum_{(r, s) \in \mathbb{Z}_{+} \oplus \mathbb{Z}_{+}} a_{r s}(r, s)=(i, j)\right\} .
$$

We will use the notation $|a|=\sum a_{r s}, a !=\prod a_{r s} !$ 


\subsection{Replication}

In this section we show that the formulas for the graded traces of elements of $M$ acting on $V^{\natural}$ (i.e., the McKay-Thompson series) are replicable [8], in fact, we show that they are completely replicable in the sense of [27] (see also [9] and [10]). We also observe that they can be computed recursively, as in [4]. The free Lie algebra structure of $\mathfrak{u}^{-}$allows us to perform a calculation analogous to one appearing in [6], computing the dimensions of homogeneous subspaces of a free Lie algebra. In this context the replication formulas occur quite naturally.

Recall the structure of $\mathfrak{u}^{-}$and $\mathcal{V}=H_{1}\left(\mathfrak{u}^{-}\right)$as $\mathbb{Z} \oplus \mathbb{Z}$-graded $M$-modules $((9)$, Lemma 2.2, Lemma 2.3, (18)). We index the grading by $p^{-1}$ and $q^{-1}$ as in (28); then write $\mathfrak{u}^{-}$and $\mathcal{V}=H_{1}\left(\mathfrak{u}^{-}\right)$as elements of $R[M][[p, q]]$ :

$$
\mathfrak{u}^{-}=\sum_{(m, n)} V_{m n}^{\natural} p^{m} q^{n}
$$

and

$$
\mathcal{V}=\sum_{(m, n)} V_{m+n-1}^{\natural} p^{m} q^{n},
$$

where here and below the sums are over all pairs $(m, n)$ such that $m, n>0$.

Define

$$
H_{t}\left(\mathfrak{u}^{-}\right)=\sum_{i=0}^{\infty} H_{i}\left(\mathfrak{u}^{-}\right) t^{i}
$$

and let $H\left(\mathfrak{u}^{-}\right)$denote the alternating sum $\left.H_{t}\left(\mathfrak{u}^{-}\right)\right|_{t=-1}$. Recall the EulerPoincaré identity:

$$
\bigwedge_{-1}\left(\mathfrak{u}^{-}\right)=H\left(\mathfrak{u}^{-}\right) .
$$

Taking log of both sides of (34) results in the formal power series identity in $R(M)[[p, q]] \otimes \mathbb{Q}:$

$$
\log \bigwedge_{-1}\left(\mathfrak{u}^{-}\right)=\log H\left(\mathfrak{u}^{-}\right)
$$

where we have

$$
\log H\left(\mathfrak{u}^{-}\right)=\log \left(1-H_{1}\left(\mathfrak{u}^{-}\right)\right)=-\sum_{n=1}^{\infty} \frac{1}{n} H_{1}\left(\mathfrak{u}^{-}\right)^{n} .
$$

Formally integrating (22), with $W=\mathfrak{u}^{-}$, gives

$$
\log \bigwedge_{t}\left(\mathfrak{u}^{-}\right)=-\sum_{n \geq 0} \Psi^{n+1}\left(\mathfrak{u}^{-}\right) \frac{(-t)^{n+1}}{n+1} .
$$

Then setting $t=-1$ gives:

$$
-\log \bigwedge_{-1}\left(\mathfrak{u}^{-}\right)=\sum_{k=1}^{\infty} \frac{1}{k} \Psi^{k}\left(\mathfrak{u}^{-}\right) .
$$


Since $H_{1}\left(\mathfrak{u}^{-}\right)=\mathcal{V}$, equation (35) and equations (32) and (33) imply

$$
\begin{gathered}
\sum_{k=1}^{\infty} \frac{1}{k} \Psi^{k}\left(\sum_{(m, n)} V_{m n}^{\natural} p^{m} q^{n}\right)=\sum_{k=1}^{\infty} \frac{1}{k}\left(\sum_{(m, n)} V_{m+n-1}^{\natural} p^{m} q^{n}\right)^{k} \\
\sum_{k=1}^{\infty} \sum_{(m, n)} \frac{1}{k} \Psi^{k}\left(V_{m n}^{\natural}\right) p^{m k} q^{n k}=\sum_{k=1}^{\infty} \frac{1}{k} \sum_{\substack {(i, j) \in \mathbb{Z}_{+} \\
\begin{subarray}{c}{a \in P(i, j) \\
|a|=k{ ( i , j ) \in \mathbb { Z } _ { + } \\
\begin{subarray} { c } { a \in P ( i , j ) \\
| a | = k } }\end{subarray}} \frac{|a| !}{a !} \prod_{r, s \in \mathbb{Z}_{+}}\left(V_{r+s-1}^{\natural}\right)^{a_{r s}} p^{i} q^{j} \\
\sum_{(i, j)} \sum_{k \mid(i, j)} \frac{1}{k} \Psi^{k}\left(V_{i j / k^{2}}^{\natural}\right) p^{i} q^{j}=\sum_{(i, j)} \sum_{a \in P(i, j)} \frac{(|a|-1) !}{a !} \prod_{r, s \in \mathbb{Z}_{+}}\left(V_{r+s-1}^{\natural}\right)^{a_{r s}} p^{i} q^{j} .
\end{gathered}
$$

Take the trace of an element $g \in M$ on both sides of this formula, writing $c_{g}(m)$ for $\operatorname{Tr}\left(g \mid V_{m}\right)$ :

$$
\sum_{(i, j)} \sum_{k \mid(i, j)} \frac{1}{k} \Psi^{k}\left(c_{g}\left(i j / k^{2}\right)\right) p^{i} q^{j}=\sum_{(i, j)} \sum_{a \in P(i, j)} \frac{(|a|-1) !}{a !} \prod_{r, s \in \mathbb{Z}_{+}} c_{g}(r+s-1)^{a_{r s}} p^{i} q^{j} .
$$

Equating the coefficients of $p^{i} q^{j}$ gives

$$
H_{i, j}=\sum_{k \mid(i, j)} \frac{1}{k} H_{i j / k^{2}}^{(k)}
$$

where, using the notation of [27], we set (for each $g \in M$ )

$$
H_{i}^{(k)}=c_{g^{k}}(i)
$$

and

$$
H_{i, j}=\sum_{a \in P(i, j)} \frac{(|a|-1) !}{a !} \prod_{r, s \in \mathbb{Z}_{+}} c_{g}(r+s-1)^{a_{r s}} .
$$

Formula (36) asserts precisely that the McKay-Thompson series

$$
T_{g}\left(V^{\natural}\right)=\sum_{i \in \mathbb{Z}} c_{g}(i) p^{i},
$$

$g \in M$, satisfy the "replication formula" as it is written in [27] (see also [8], [9] and $[10])$.

Recall the definition of "replicability" given in [27]: that $H_{i, j}=H_{m, n}$ whenever $i j=m n$ and $\operatorname{gcd}(i, j)=\operatorname{gcd}(m, n)$. (See also [9], where this definition is related to the replication formulas of [8].) Since all of the "replicates" $T_{g^{k}}\left(V^{\natural}\right)$ of the McKay-Thompson series $T_{g}\left(V^{\natural}\right)$ are replicable, the $T_{g}\left(V^{\natural}\right)$ are "completely replicable" ([27]; see also [10]). Thus we observe from (36): 
Proposition 5.2 The McKay-Thompson series $T_{g}\left(V^{\natural}\right)=\sum_{i \in \mathbb{Z}} c_{g}(i) p^{i}, g \in$ $M$, are completely replicable functions.

The modular functions of [8] also satisfy (36), by [27], [23], [9] and [10].

In order to obtain recursion relations for the McKay-Thompson series, we apply Möbius inversion to (36), with the functions $t((i, j))=(i+j) c_{g}(i j)$ and $s((i, j))=(i+j) H_{i, j}$ :

$$
\begin{gathered}
c_{g}(i j)=\sum_{\substack{k>0 \\
k(m, n)=(i, j)}} \frac{1}{k} \mu(k) \Psi^{k}\left(\sum_{a \in P(m, n)} \frac{(|a|-1) !}{a !} \prod_{r, s \in \mathbb{Z}_{+}} c_{g}(r+s-1)^{a_{r s}}\right) \\
=\sum_{\substack{k>0 \\
k(m, n)=(i, j)}} \frac{1}{k} \mu(k)\left(\sum_{a \in P(m, n)} \frac{(|a|-1) !}{a !} \prod_{r, s \in \mathbb{Z}_{+}} c_{g^{k}}(r+s-1)^{a_{r s}}\right) .
\end{gathered}
$$

If $Q$ is a polynomial expression in the $c_{g}(n), g \in M, n \leq N$ we say that $Q$ has level $\leq N$. Then (37) implies that

$$
c_{g}(i j)=c_{g}(i+j-1)+\text { an expression of level } \leq i+j-3 .
$$

This observation is, of course, vacuous if $i$ or $j=1$. Taking $(i, j)=(2 l, 2)$ equation (38) gives

$$
c_{g}(4 l)=c_{g}(2 l+1)+\text { an expression of level } \leq 2 l-1 .
$$

Taking $(i, j)=(2 l+1,2)$ equation $(38)$ gives

$$
c_{g}(4 l+2)=c_{g}(2 l+2)+\text { an expression of level } \leq 2 l .
$$

For $(i, j)=(l, 4)$ equation $(38)$ gives

$$
c_{g}(4 l)=c_{g}(l+3)+\text { an expression of level } \leq l+1 .
$$

It follows that the value of $c_{g}(4 l)$ is given by an expression of lower level whenever $4 l>2 l+1$, i.e., for $l \geq 1$. Also, $c_{g}(4 l+2)$ is given by an expression of lower level whenever $4 l+2>2 l+2$, i.e., for $l \geq 1$. Finally, equating the two expressions for $c_{g}(4 l)$ shows that $c_{g}(2 l+1)$ is given by an expression of lower level whenever $2 l+1>l+3$, i.e., for $l>2$. Thus $c_{g}(n)$ is determined by expressions of lower level except when $n=1,2,3,5$. Thus the values of the $c_{g}(n)$ are determined by the $c_{h}(1), c_{h}(2), c_{h}(3), c_{h}(5), h \in M$, and equation (37).

As in [4], we conclude that since both the McKay-Thompson series and the modular functions of [8] satisfy (37), all that is necessary to prove that these functions are the same (see [4]) is to check the initial data listed above. 


\section{References}

[1] M. Atiyah, K-Theory, W.A. Benjamin, New York, 1967.

[2] R. Borcherds, Generalized Kac-Moody Lie algebras, J. Algebra 115 (1988), 501-512.

[3] R. Borcherds, Central extensions of generalized Kac-Moody Lie algebras, $J$. Algebra 140 (1991), 330-335.

[4] R. Borcherds, Monstrous moonshine and monstrous Lie superalgebras, Invent. Math. 109 (1992), 405-444.

[5] R. Borcherds, Sporadic groups and string theory, to appear.

[6] N. Bourbaki, Lie Groups and Lie Algebras, Part 1, Hermann, Paris, 1975.

[7] H. Cartan and S. Eilenberg, Homological Algebra, Princeton University Press, 1956.

[8] J. H. Conway and S. P. Norton, Monstrous Moonshine, Bull. London Math. Soc. 11 (1979), 308-339.

[9] C. J. Cummins and S. P. Norton, Rational Hauptmoduls are replicable, to appear.

[10] C. Ferenbaugh, Replication formulae for Monster-like functions, to appear.

[11] I. Frenkel, J. Lepowsky and A. Meurman, A natural representation of the Fischer-Griess Monster with the modular function $J$ as character, Proc. Natl. Acad. Sci. USA 81 (1984), 3256-3260.

[12] I. Frenkel, J. Lepowsky and A. Meurman, Vertex Operator Algebras and the Monster, Academic Press, Boston, 1988.

[13] O. Gabber and V. Kac, On defining relations of certain infinite-dimensional Lie algebras, Bull. Amer. Math. Soc. 5 (1981), 185-189.

[14] H. Garland and J. Lepowsky, Lie algebra homology and the Macdonald-Kac formulas, Invent. Math. 34 (1976), 37-76.

[15] R. Gebert and J. Teschner, On the fundamental representation of Borcherds algebras with one imaginary simple root, Lett. Math. Phys, to appear.

[16] P. Goddard and C. Thorn, Compatability of the dual Pomeron with unitarity and the absence of ghosts in the dual resonance model, Phys. Lett. 43 No. 2 (1972), 235-238.

[17] K. Harada, M. Miyamoto and H. Yamada, A generalization of Kac-Moody Lie algebras, to appear. 
[18] E. Jurisich, Generalized Kac-Moody algebras and their relation to free Lie algebras, Ph.D. thesis, Rutgers University, May, 1994.

[19] E. Jurisich, Generalized Kac-Moody Lie algebras, free Lie algebras and the structure of the Monster Lie algebra, J. Pure and Applied Algebra, to appear.

[20] V. Kac, Simple irreducible graded Lie algebras of finite growth, Math. USSR Izvestija 2 (1968), 1271-1311.

[21] V. Kac, Infinite Dimensional Lie Algebras, Cambridge University Press, third edition, 1990.

[22] D. Knutson, $\lambda$-Rings and the Representation Theory of the Symmetric Group, Lecture Notes in Mathematics 308, Springer-Verlag, 1973.

[23] M. Koike, On replication formula and Hecke operators, preprint.

[24] J. Lepowsky, Lectures on Kac-Moody Lie algebras, Université Paris VI, 1978 (unpublished).

[25] J. Lepowsky, Generalized Verma modules, loop space cohomology and Macdonald-type identities, Ann. Scient. Ec. Norm. Sup., 4 série 12 (1979), 169-234.

[26] R. Moody, A new class of Lie algebras, J. Algebra 10 (1968), 211-230.

[27] S. P. Norton, More on Moonshine, in: Computational Group Theory, Academic Press, London, 1984, 185-193.

[28] J.-P. Serre, Lie Algebras and Lie Groups, W.A. Benjamin, New York, 1965.

[29] J.-P. Serre, Algèbres de Lie semi-simples complexes, W.A. Benjamin, New York, 1966. 